\title{
Chemistry and Molecular Dynamics Simulations of Heme b-HemQ and Coproheme-HemQ
}

\author{
Stefan Hofbauer, ${ }^{\dagger}$ Marco Dalla Sega, ${ }^{\ddagger}$ Stefan Scheiblbrandner, ${ }^{\S}$ Zuzana Jandova, ${ }^{\ddagger}$ Irene Schaffner, \\ Georg Mlynek, ${ }^{\dagger}$ Kristina Djinović-Carugo, ${ }^{\dagger, \#}$ Gianantonio Battistuzzi, ${ }^{\perp}$ Paul G. Furtmüller, \\ Chris Oostenbrink, and Christian Obinger*,
}

${ }^{\dagger}$ Department for Structural and Computational Biology, Max F. Perutz Laboratories, University of Vienna, A-1030 Vienna, Austria ${ }^{\ddagger}$ Department of Material Sciences and Process Engineering, Institute of Molecular Modeling and Simulation, BOKU-University of Natural Resources and Life Sciences, A-1190 Vienna, Austria

${ }^{\S}$ Department of Food Science and Technology, Food Biotechnology Laboratory, BOKU-University of Natural Resources and Life Sciences, A-1190 Vienna, Austria

"Department of Chemistry, Division of Biochemistry, VIBT-Vienna Institute of BioTechnology, BOKU-University of Natural Resources and Life Sciences, A-1190 Vienna, Austria

${ }^{\perp}$ Department of Chemistry and Geology, University of Modena and Reggio Emilia, 41125 Modena, Italy

\#Department of Biochemistry, Faculty of Chemistry and Chemical Technology, University of Ljubljana, 1000 Ljubljana, Slovenia

\section{Supporting Information}

\begin{abstract}
Recently, a novel pathway for heme $b$ biosynthesis in Gram-positive bacteria has been proposed. The final poorly understood step is catalyzed by an enzyme called HemQ and includes two decarboxylation reactions leading from coproheme to heme $b$. Coproheme has been suggested to act as both substrate and redox active cofactor in this reaction. In the study presented here, we focus on HemQs from Listeria monocytogenes (LmHemQ) and Staphylococcus aureus (SaHemQ) recombinantly produced as apoproteins in Escherichia coli. We demonstrate the rapid and two-phase uptake of coproheme by both apo forms and the significant differences in thermal stability of the apo forms, coprohemeHemQ and heme $b$-HemQ. Reduction of ferric high-spin coproheme-HemQ to the ferrous form is shown to be enthalpically favored but entropically disfavored with standard reduction potentials of $-205 \pm 3 \mathrm{mV}$ for LmHemQ and $-207 \pm 3 \mathrm{mV}$ for $\mathrm{SaHemQ}$ versus the standard hydrogen electrode at $\mathrm{pH}$ 7.0. Redox thermodynamics suggests the presence of a pronounced H-bonding network and restricted solvent mobility in the heme cavity. Binding of cyanide to the sixth coproheme position is monophasic but relatively slow $\left(\sim 1 \times 10^{4} \mathrm{M}^{-1}\right.$ $\mathrm{s}^{-1}$ ). On the basis of the available structures of apo-HemQ and modeling of both loaded forms, molecular dynamics simulation allowed analysis of the interaction of coproheme and heme $b$ with the protein as well as the role of the flexibility at the proximal heme cavity and the substrate access channel for coproheme binding and heme $b$ release. Obtained data are discussed with respect to the proposed function of HemQ in monoderm bacteria.
\end{abstract}

HemQ is an enzyme involved in the late stages of the heme biosynthetic pathway of monoderm (Gram-positive) bacteria. ${ }^{1-3}$ It catalyzes the decarboxylation of iron coproporphyrin III (coproheme) to yield heme $b$ via an unusual peroxidedependent reaction that is poorly understood. ${ }^{4}$ HemQs are structurally and phylogenetically related to two families of heme enzymes, i.e., chlorite dismutases (Cld) and dye-decolorizing peroxidases (DyP), and were originally designated in the literature as Cld-like proteins (Figure 1A,B).$^{5-7}$ However, the functions of $\mathrm{Cld}, \mathrm{DyP}$, and HemQ are very diverse and specialized.

Clds are oxidoreductases that are able to convert chlorite into chloride and dioxygen. ${ }^{8}$ In this reaction, an oxygen-oxygen double bond is formed, a reaction so far described only for the manganese water-splitting complex of Photosystem II. ${ }^{9}$ DyPs are versatile peroxidases, capable of performing hydrogen peroxide-dependent one-electron oxidations of various aromatic compounds. ${ }^{10,11}$ In both Clds and DyPs, a heme $b$ is tightly bound to the protein by a proximal histidine, which is part of an extended H-bonding network..$^{5,12-16}$ In addition, Clds have a catalytic distal arginine, ${ }^{5,17}$ which is important for stabilization of the transiently produced hypochlorite during the reaction. ${ }^{18,19}$ In DyPs, the catalytic distal arginine structurally aligns with the distal arginine of Clds, but in addition, a catalytic aspartate is present. ${ }^{20-23}$

Received: July 11, 2016

Revised: August 22, 2016

Published: September 6, 2016 


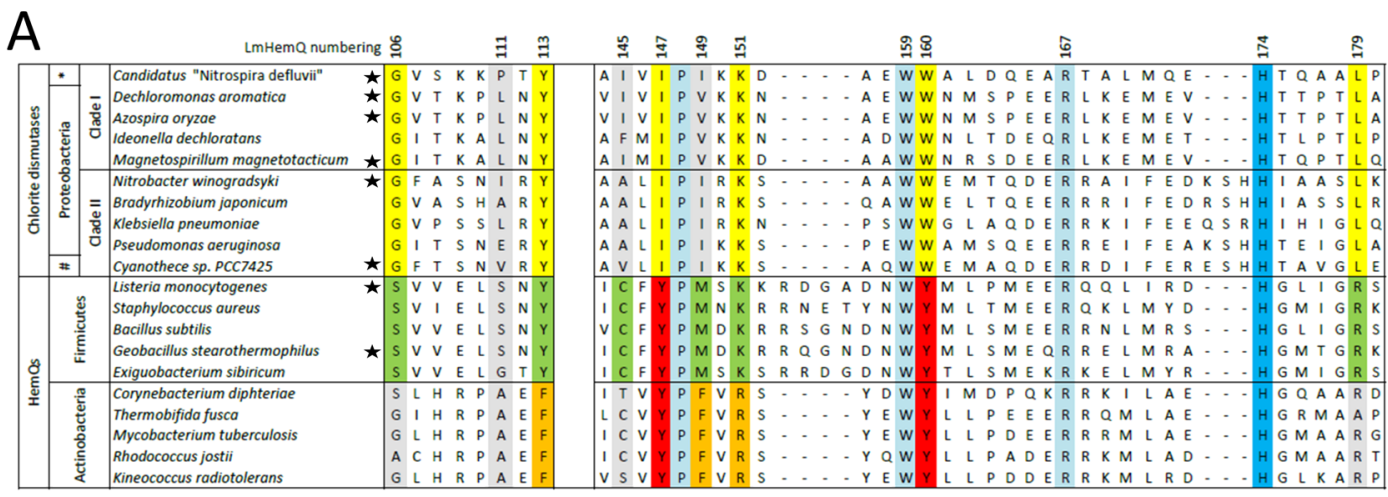

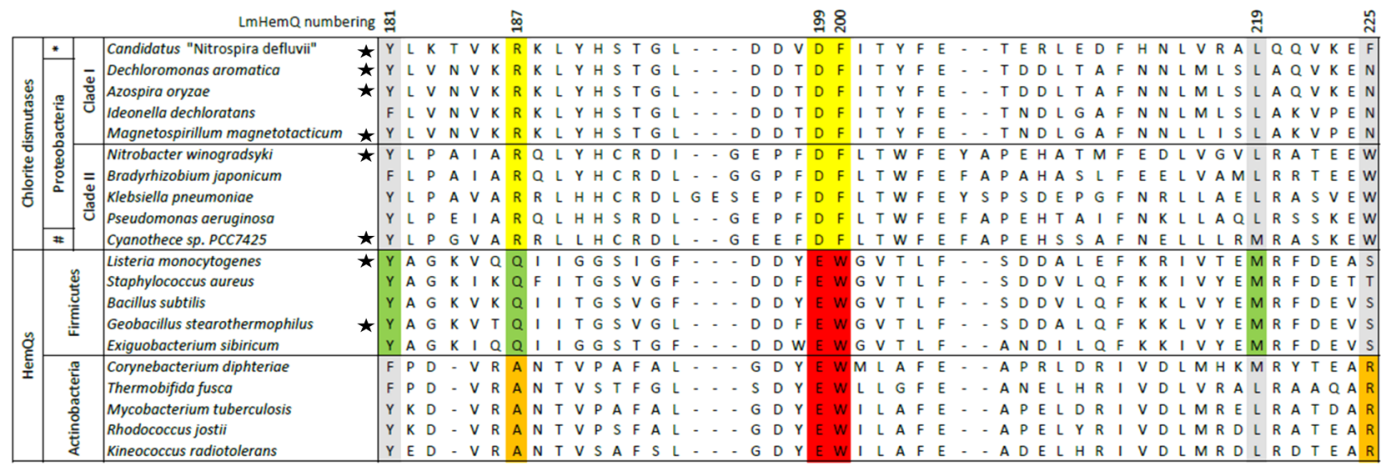

* Nitrospira

\# Cyanobacterium

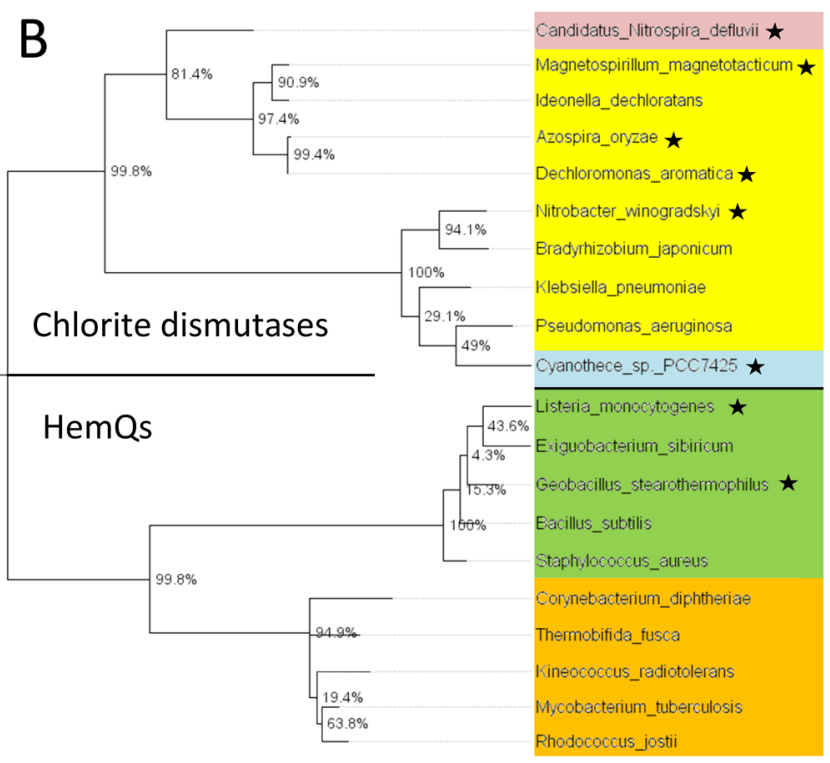

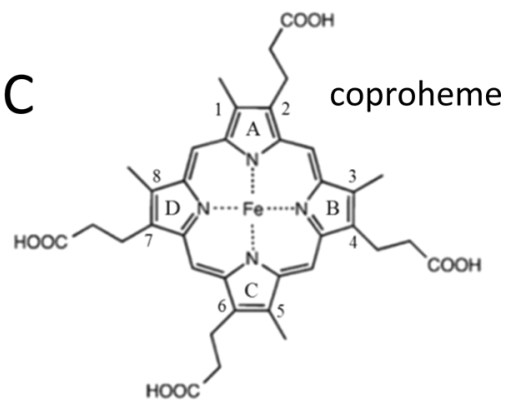

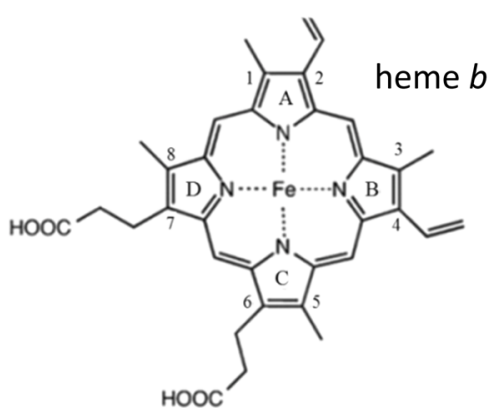

Figure 1. Sequence analysis and phylogenetics of HemQs and chlorite dismutases. (A) Sequence alignment of selected chlorite dismutase (Cld) and HemQ sequences. Completely conserved residues are highlighted in blue background (proximal histidine in dark blue); relevant conserved residues are highlighted in yellow for Cld, red for HemQs, green for HemQs from Firmicutes, and orange for Actinobacteria. (B) Phylogenetic tree (maximum likelihood) of Clds and HemQs based on the sequence alignment presented in panel A. Proteins from proteobacteria, Firmicutes, Actinobacteria, cyanobacteria, and Nitrospira are highlighted in yellow, green, orange, blue, and red, respectively. Asterisks indicate available crystal structures deposited in the Protein Data Bank. (C) Comparison of structures of coproheme and heme $b$.

HemQs were demonstrated to bind heme $b$ reversibly with low affinities, and no substantial enzymatic activities toward hydrogen peroxide or chlorite could be detected for heme $b$ bound HemQs. ${ }^{24-26}$ Similar to the case in Cld and DyP, in HemQ the proximal ligand is also a histidine (H174, LmHemQ numbering), but the lack of the proximal hydrogen bonding network seems to be responsible for its weak heme $b$ binding properties. $^{27}$ In contrast to Clds and DyPs, no charged amino acid residue is found distally of the predicted heme binding site. On the basis of structural and sequence alignments, a glutamine is present in HemQs from Firmicutes (Q187) and an alanine in Actinobacteria at the respective position of the catalytic arginine in Clds or DyPs. This might explain the absence of any significant catalase, peroxidase, or chlorite dismutase 
activity. ${ }^{27}$ Until now, there have been only apo structures of HemQs available in the Protein Data Bank (PDB) (entries 4WWS and 1T0T).

Dailey and co-workers discovered that HemQ is an essential enzyme in the heme biosynthesis of Firmicutes and Actinobacteria and identified coproheme as its substrate. ${ }^{1,24}$ Coproheme has four propionate groups located at positions $2,4,6$, and 7 of the porphyrin ring. Propionates at positions 2 and 4 are decarboxylated by HemQ in a stepwise fashion to form the respective vinyl groups of heme $b$ (Figure 1C). ${ }^{1}$ The interactions of coproheme with the protein moiety of HemQ as well as the catalytic reaction mechanism of the decarboxylation reactions are unknown. HemQs are attractive targets for the development of new classes of therapeutics, because many organisms with coding sequences for HemQ are pathogens (e.g., Listeria monocytogenes, Staphylococcus aureus, and Mycobacterium tuberculosis); several have developed antibiotic resistances and are a major threat. The equilibrium established by the organisms of iron uptake, heme biosynthesis, heme degradation, and iron release is very sensitive and essential for their viability. ${ }^{28}$

To understand the enzymology of HemQ, we need to characterize the interactions of coproheme and heme $b$ with HemQ at the biochemical and biophysical level to serve as a starting point for the elucidation of the reaction mechanism and the identification of intermediate redox species. Here we report the biochemical and biophysical properties of coprohemeHemQs from L. monocytogenes and S. aureus, including (i) spectroscopic characterization, (ii) the kinetics of binding of coproheme and hemin to the apo forms, (iii) the kinetics of binding of cyanide to coproheme-HemQ and heme $b$-HemQ and (iv) the redox thermodynamics of the $\mathrm{Fe}(\mathrm{III}) / \mathrm{Fe}$ (II) couple. Moreover, we present molecular dynamics simulation data of a coproheme-HemQ model that allow us to discuss the structural differences among apo-HemQ coproheme-HemQ and heme $b$-HemQ, providing an important basis for the elucidation of the mechanism of conversion of coproheme to heme $b$.

\section{MATERIALS AND METHODS}

Phylogenetic Analysis. A selection of $120 \mathrm{Cld}$ and HemQ sequences were collected from public databases (Uniprot, NCBI). First, multiple-sequence alignments for Clds and HemQs were constructed using MUSCLE ${ }^{29}$ with the following parameters: gap penalties, -2,9; gap extension, 0; hydrophobicity multiplier, 1.2 ; maximum number of iterations, 8 . From these sequence alignments, a phylogenetic tree of Cld and HemQ proteins was reconstructed with the maximum likelihood algorithm using the Jones-Taylor-Thornton (JTT) model, with the $\gamma$ parameter set to 3 and 1000 bootstrap replications; complete deletion was used for gaps and/or missing data treatment. From the original sequences, 20 were selected for presentation in this work, covering subfamily I and subfamily II of the Clds and both subfamilies of confirmed HemQs (Firmicutes and Actinobacteria). All tools for sequence alignments and phylogenetic tree reconstruction were embedded in the MEGA5 package. ${ }^{30}$ The phylogenetic tree was drawn with FigTree version 1.4 (http://tree.bio.ed.ac.uk/software/ figtree/).

Expression and Purification of HemQs. Cloning, expression, and purification of the apo form of HemQ from L. monocytogenes (LmHemQ) were described previously. ${ }^{26}$ We kindly received the plasmid for HemQ from $S$. aureus
(SaHemQ) from L. M. Saraiva [Universidade Nova de Lisboa (ITQB), Lisbon, Portugal]. Cloning, expression, and purification were described recently. ${ }^{3}$ Briefly, SaHemQ was cloned into a pET-23b vector, with a C-terminal poly-His tag. Heterologous expression was performed at $16{ }^{\circ} \mathrm{C}$ overnight with $180 \mathrm{rpm}$ shaking (after induction at an $\mathrm{OD}_{600}$ of approximately 0.6 ) in Escherichia coli BL21(DE3)pLysS cells (Merck/Novagen). Purification of the apoprotein was performed by affinity chromatography using a HisTrap column (GE Healthcare). Apo-HemQ was stored at $-80{ }^{\circ} \mathrm{C}$ and, if needed, reconstituted with equimolar concentrations coproheme (Frontiers Scientific) or hemin (Sigma) to yield holo-HemQ. All protein concentrations of pentameric HemQs refer to subunit concentrations.

Kinetics of Binding of Coproheme to HemQ and Cyanide to Coproheme-HemQ. Time-resolved binding of coproheme to apo-HemQ was monitored using a stopped-flow apparatus equipped with a diode array detector (model SX$18 \mathrm{MV}$, Applied Photophysics), in the conventional mode. The optical quartz cell with a path length of $10 \mathrm{~mm}$ had a volume of $20 \mu \mathrm{L}$. The fastest mixing time was $1 \mathrm{~ms}$. All measurements were performed at $25{ }^{\circ} \mathrm{C}$. Typically, the concentration of coproheme in the cell was $0.25-1.5 \mu \mathrm{M}$, and HemQ was present in excess, to ensure pure spectral species of the coproheme-bound proteins. Experiments were conducted in 50 $\mathrm{mM}$ phosphate buffer ( $\mathrm{pH} 7.0)$. Reactions were simulated, and rates were estimated using Pro-Kineticists software (Applied Photophysics). The apparent second-order rate constants, $k_{\text {on }}$ were obtained from the slope of a plot of $k_{\mathrm{obs}}$ versus coproheme concentration.

For the studies of binding of cyanide to ferric coprohemeLmHemQ and coproheme-SaHemQ the increase in absorbance at $410 \mathrm{~nm}$ was monitored. In a typical experiment, one syringe contained $2 \mu \mathrm{M}$ HemQ in $50 \mathrm{mM}$ buffered solution ( $\mathrm{pH} \mathrm{5.5-10.0)} \mathrm{and} \mathrm{the} \mathrm{second} \mathrm{syringe} \mathrm{contained} \mathrm{an} \mathrm{at} \mathrm{least} \mathrm{5-}$ fold excess of cyanide in the same buffer. A minimum of three measurements were performed for each ligand concentration. Additionally, binding of cyanide to coproheme-HemQ was also investigated using the diode array detector (Applied Photophysics), which allowed the synthesis of artificial sets of timedependent spectra as well as spectral analysis of enzyme intermediates.

Heme Transfer. Transfer of the prosthetic group from HemQ to apo-myoglobin (apo-Mb) was followed photometrically over time. Apo-Mb (horse heart, Sigma) was prepared by a modified extraction method of Teale, as described previously. ${ }^{26,31,32}$ Experiments were performed using a Specord S10 UV-vis diode array spectrophotometer (Zeiss) in the cyclic measurement mode under constant stirring, which allows time-resolved monitoring over time of the entire UV-vis absorption spectrum $(200-800 \mathrm{~nm})$. Typically, $1 \mu \mathrm{M}$ coproheme- or heme $b$-bound LmHemQ or SaHemQ was incubated with a 10 -fold excess of apo- $\mathrm{Mb}$ in $50 \mathrm{mM}$ phosphate buffer ( $\mathrm{pH}$ 7.0). For comparison, binding of $1 \mu \mathrm{M}$ coproheme or $1 \mu \mathrm{M}$ hemin to $10 \mu \mathrm{M}$ apo-Mb was also tested.

Thermal Stability of Apo- and Holo-HemQ. Differential scanning calorimetry (DSC) measurements were performed using a VP-capillary DSC microcalorimeter from Microcal with a cell volume of $137 \mu \mathrm{L}$. The measurements were controlled by the VP-viewer program, and the instrument was equipped with an autosampler for 96-well plates. Samples were analyzed using a programmed heating scan rate of $60{ }^{\circ} \mathrm{C} \mathrm{h}^{-1}$ over a temperature range from 20 to $100{ }^{\circ} \mathrm{C}$, and the cell pressure 
was approximately $60 \mathrm{psi}$ (4.136 bar). DSC thermograms were corrected for buffer baseline and protein concentration. Apo-, heme $b$-, or coproheme-LmHemQ and SaHemQ (5 $\mu \mathrm{M}$ each) in $50 \mathrm{mM}$ phosphate buffer $(\mathrm{pH} 7.0)$ were used for each measurement. For data analysis and conversion, the Microcal origin software was used. Heat capacity $\left(C_{p}\right)$ was expressed in kilocalories per mole per kelvin. Data points were fitted to nontwo-state equilibrium unfolding models by the LavenbergMarquardt (LM) nonlinear least-squares method.

UV-Visible Electronic Absorption Spectroscopy and Electronic Circular Dichroism Spectroscopy. UV-vis spectroscopic studies were performed at $25{ }^{\circ} \mathrm{C}$ between 250 and $700 \mathrm{~nm}$ on a Hitachi U-3900 UV-vis spectrophotometer. Temperature was controlled with a water bath connected to the cuvette holder. The path length was $10 \mathrm{~mm}$, and the scan rate was $600 \mathrm{~nm} \mathrm{~min}^{-1}$. Typically, 6-15 $\mu \mathrm{M}$ enzyme (LmHemQ or SaHemQ) was measured. To obtain the ferrous form, samples were chemically reduced by addition of $10 \mathrm{mM}$ freshly prepared sodium dithionite between $\mathrm{pH} 5.5$ and 10.0 (50 mM phosphate-citrate buffer, $50 \mathrm{mM}$ phosphate buffer, or $50 \mathrm{mM}$ glycine $/ \mathrm{NaOH}$ buffer).

Electronic circular dichroism spectroscopy (ECD) was performed using Chirascan (Applied Photophysics). The instrument was flushed with a nitrogen flow of $5 \mathrm{~L} \mathrm{~min}{ }^{-1}$ that allowed the simultaneous monitoring of $\mathrm{UV}-$ vis electronic absorption and circular dichroism. Coproheme-HemQ at 10 $\mu \mathrm{M}$ was analyzed in the far-UV region $\left(190-260 \mathrm{~nm} ; 5 \mathrm{~s} \mathrm{~nm}^{-1}\right.$ scan speed, $3 \mathrm{~nm}$ bandwidth, $1 \mathrm{~mm}$ path length) and in the near-UV and visible region $\left(260-500 \mathrm{~nm} ; 5 \mathrm{~s} \mathrm{~m}^{-1}\right.$ scan speed, $1 \mathrm{~nm}$ bandwidth, $10 \mathrm{~mm}$ path length). All measurements were performed in $50 \mathrm{mM}$ phosphate buffer ( $\mathrm{pH} 7.0$ ).

Electron Paramagnetic Resonance Spectroscopy (EPR). EPR was performed on a Bruker EMX continuous wave $(\mathrm{cw})$ spectrometer, operating at X-band $(9 \mathrm{GHz})$ frequencies. The instrument was equipped with a highsensitivity resonator and an Oxford Instruments ESR900 helium cryostat for low-temperature measurements. Spectra were recorded under nonsaturating conditions using a microwave power of $2 \mathrm{~mW}$, a modulation frequency of $100 \mathrm{kHz}$, a modulation amplitude of $1 \mathrm{mT}$, a conversion time of $40 \mathrm{~ms}$, a time constant of $40 \mathrm{~ms}$, and 2048 points. Samples $(100 \mu \mathrm{L}$ of a $50 \mu \mathrm{M}$ solution) of recombinant LmHemQ and SaHemQ were prepared in $100 \mathrm{mM}$ phosphate buffer ( $\mathrm{pH} 7.0)$, transferred into Wilmad quartz tubes ( $3 \mathrm{~mm}$ inner diameter), and flashfrozen in liquid nitrogen. Cyanide binding was tested in the presence of $1 \mathrm{mM}$ cyanide and HemQ concentrations of 100 $\mu \mathrm{M}$. To remove $\mathrm{O}_{2}$, the tubes were flushed with argon while the sample was kept frozen on dry ice. Measurements were taken at $10 \mathrm{~K}$. The spectra were simulated with the Easyspin toolbox for Matlab ${ }^{33}$ and consist of a weighted sum of simulations of the individual high-spin and low-spin species. The rhombicity was obtained from $g_{x}{ }^{\text {eff }}$ and $g_{y}$ eff, and the relative intensities were calculated on the basis of the simulations, following the procedure of Aasa and Vanngard to account for the different integral intensity per unit spin of species that display different effective $g$ values (as found in low-spin and high-spin centers). ${ }^{34,35}$

Spectroelectrochemistry. All experiments were conducted in a homemade optical transparent thin-layer electrochemical (OTTLE) cell with a path length of $0.05 \mathrm{~cm}$. The three-electrode configuration consisted of a platinum gauze working electrode (Goodfellow Cambridge Ltd.), a platinum wire auxiliary electrode (Goodfellow Cambridge Ltd.), and a
$\mathrm{RE}-6 \mathrm{Ag} / \mathrm{AgCl}$ reference electrode (BASi). The reference electrode was calibrated against a saturated calomel electrode $(\mathrm{HgCl})$. All potentials are referenced to the standard hydrogen electrode (SHE, $+242 \mathrm{mV}$ ). Potentials were applied across the OTTLE cell with a Series G 300 Potentiostat/Galvanostat/ ZRA (Gamry). UV-vis spectra were recorded with an Agilent 8453 UV-vis Diode Array System (Agilent Technologies). Spectroelectrochemical experiments were performed in an $\mathrm{O}_{2}$ free environment in a thermostated glovebox (Whitley DG250) with $500 \mu \mathrm{L}$ samples containing $80 \mu \mathrm{M}$ HemQ in $100 \mathrm{mM}$ phosphate buffer ( $\mathrm{pH} 7.0)$ in the presence of various mediators: methylviologen $(200 \mu \mathrm{M})$, indigocarmin $(4 \mu \mathrm{M})$, methylene blue $(4 \mu \mathrm{M})$, and phenazine methosulfate $(4 \mu \mathrm{M})$. The Nernst plot consisted of at least seven points and was linear with a slope consistent with a one-electron reduction process. Variable-temperature experiments were performed using an isothermal cell configuration. ${ }^{36}$ Parametrization of the entropic component was possible via calculation of $\Delta S^{\prime}$ from the slope of the plot of $E^{\prime \circ}$ versus temperature. $\Delta{S^{\prime}}^{\circ}{ }_{\mathrm{rc} \text { (reaction) was }}$

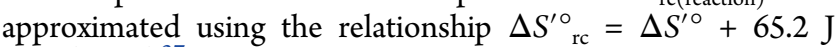
$\mathrm{mol}^{-1} \mathrm{~K}^{-1} \cdot{ }^{37} \Delta H^{\circ}{ }_{\text {rc }}$ was calculated using the following equation $^{38}$

$$
\Delta H^{\circ \prime}{ }_{\text {rc }}=-n F E^{\circ \prime}+298.16 \Delta S^{\circ \prime}{ }_{r c}
$$

Experiments with LmHemQ were performed at $20{ }^{\circ} \mathrm{C}$, and experiments with $\mathrm{SaHemQ}$ were conducted over a temperature range from 10 to $25{ }^{\circ} \mathrm{C}$.

Molecular Dynamics Simulations. Molecular dynamics simulations were performed for LmHemQ to study how coproheme fits inside the protein and to determine which residues are most likely involved in the interactions. For the simulations, we have used the GROMOS11 software package with the GROMOS 54A7 force field. ${ }^{39,40}$ Force-field parameters for the coproheme in the ferric form were derived from the heme $b$ parameters described by Zou et al. ${ }^{41}$

The pentameric LmHemQ crystal structure ${ }^{26}$ (PDB entry 4WWS) was used, modeling the missing residues (V5, K6, and N112-D122) of chains B-D by superimposing chain A on them. The coproheme molecule was built by adding two propionate groups to the porphyrin system taken from the crystal structure of the closely related chlorite dismutase (Cld) (PDB entry $3 \mathrm{NN} 1$ ). ${ }^{5}$ Because of the high degree of similarity of HemQ with Cld, the coprohemes were placed into the putative active sites according to the corresponding binding pose of heme $b$ in Cld, manually moving them slightly closer to $\mathrm{H} 174$ to facilitate coordination of iron to the $\mathrm{N}_{\varepsilon}$ atom of H174. The protein was solvated with SPC water molecules ${ }^{42}$ in a periodic rectangular box of roughly $9.4 \mathrm{~nm} \times 10.8 \mathrm{~nm} \times 11.3 \mathrm{~nm}$, together with 65 sodium ions to ensure a zero net charge for the whole system at $\mathrm{pH} 7$.

The long-range interactions were calculated employing the reaction-field scheme with a cutoff of $1.4 \mathrm{~nm}$ and a relative dielectric constant of 61 (as appropriate for the SPC model for water). ${ }^{43,44}$ Short-range interactions up to $0.8 \mathrm{~nm}$ were calculated for every time step from a pairlist that was determined every five steps. Intermediate-range interactions up to $1.4 \mathrm{~nm}$ were computed at pairlist updates and kept constant between the updates.

An equilibration phase was conducted for 20 ps, starting from $60 \mathrm{~K}$, keeping the solute positionally restrained. Four subsequent equilibration steps were performed, increasing the temperature by $60 \mathrm{~K}$ in each step and relaxing the positional restraints during heating. In the last equilibration step, the 

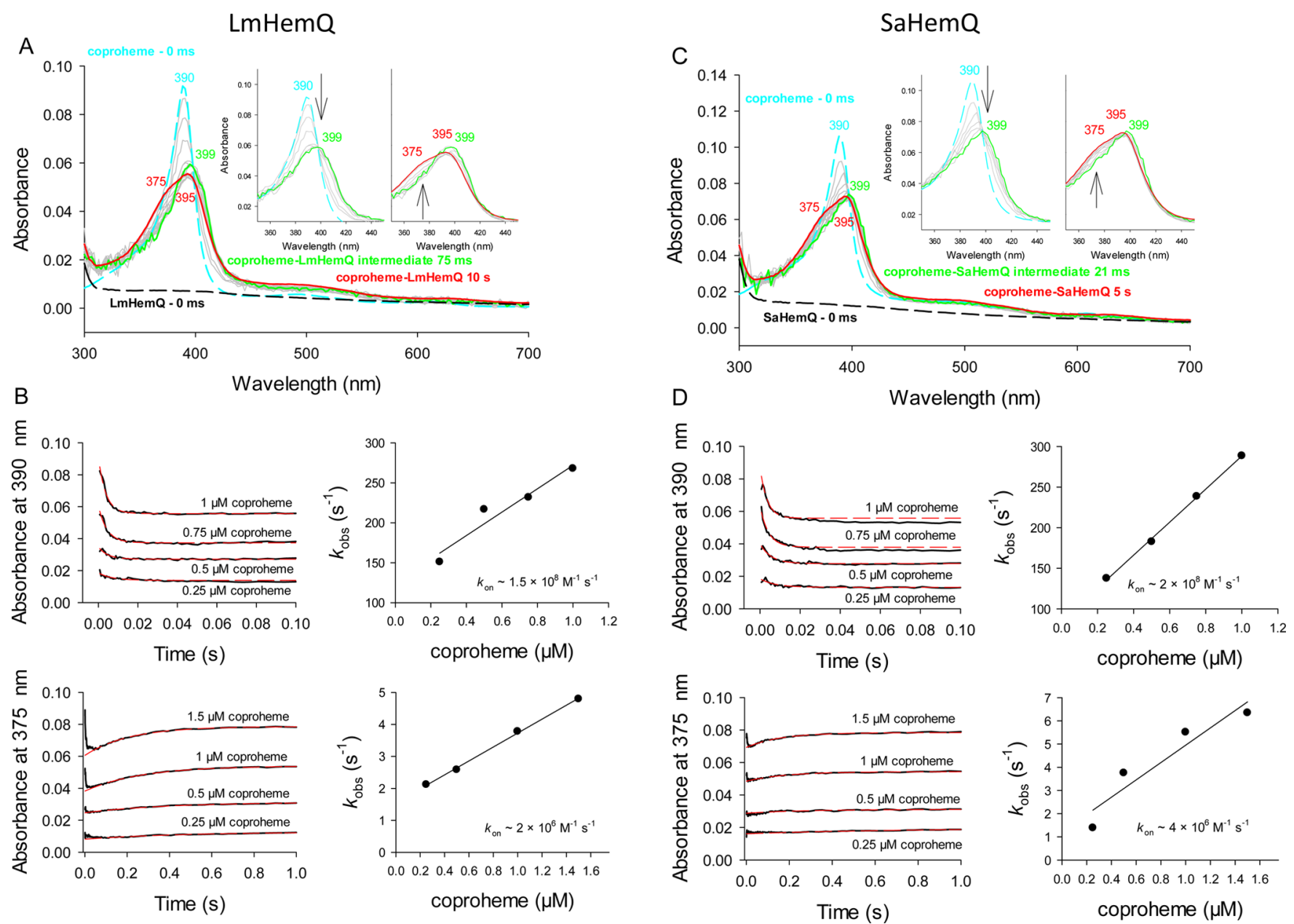

Figure 2. Kinetics of binding of coproheme to apo-HemQs from L. monocytogenes and S. aureus. (A) Spectral transitions upon binding of $1 \mu \mathrm{M}$ coproheme to $3 \mu \mathrm{M}$ apo-LmHemQ. The spectrum of apo-LmHemQ is shown as a dashed black line and that of free coproheme as a dashed cyan line. Spectra of a rapidly formed intermediate $(75 \mathrm{~ms}$ ) and of coproheme-LmHemQ (final form, $10 \mathrm{~s}$ ) are shown as solid green and red lines, respctively. In the inset, the reaction is dissected into two kinetically separated phases, i.e., rapid formation of intermediate species (spectra recorded $1,5,15,35,45,55,65$, and $75 \mathrm{~ms}$ after mixing) and slow conversion to coproheme-LmHemQ (spectra recorded $85 \mathrm{~ms}, 128 \mathrm{~ms}, 254 \mathrm{~ms}, 483 \mathrm{~ms}, 899$ $\mathrm{ms}, 3.4 \mathrm{~s}, 5.6 \mathrm{~s}$, and $10 \mathrm{~s}$ after mixing). (C) Spectral transitions upon binding of $1 \mu \mathrm{M}$ coproheme to $5 \mu \mathrm{M}$ apo-SaHemQ. The spectrum of apo$\mathrm{SaHemQ}$ is shown as a dashed black line and that of free coproheme as a dashed cyan line. Spectra of a rapidly formed intermediate (21 ms) and of coproheme-LmHemQ (final form, $5 \mathrm{~s}$ ) are shown as solid green and red lines, respectively. In the inset, the reaction is dissected into two kinetically separated phases, i.e., rapid formation of intermediate species (spectra recorded 1, 3, 4, 6, 10, and $21 \mathrm{~ms}$ after mixing) and slow conversion to coproheme-SaHemQ (spectra recorded $29 \mathrm{~ms}, 49 \mathrm{~ms}, 101 \mathrm{~ms}, 189 \mathrm{~ms}, 338 \mathrm{~ms}, 592 \mathrm{~ms}, 1 \mathrm{~s}, 1.8 \mathrm{~s}, 3 \mathrm{~s}$, and $5 \mathrm{~s}$ after mixing). Conditions: $50 \mathrm{mM}$ phosphate buffer ( $\mathrm{pH} 7.0$ ). (B and D) Time traces followed at $390 \mathrm{~nm}$ (fast phase) and $375 \mathrm{~nm}$ (slow phase) derived from stopped-flow experiments (left panels): black lines for experimental time traces and red dashed lines for single-exponential fits of time traces. The corresponding plots of calculated $k_{\mathrm{obs}}$ values vs ligand concentration are shown at the right.

system was propagated for 300 ps. A production run was then performed for $30 \mathrm{~ns}$ using a Berendsen thermostat and barostat ${ }^{45}$ with coupling times of 0.1 and 0.5 ps to keep the temperature and pressure constant at $300 \mathrm{~K}$ and 1 atm, respectively. The isothermal compressibility was set to $4.575 \times$ $10^{-4} \mathrm{~kJ}^{-1} \mathrm{~mol} \mathrm{~nm}$. Simulations were conducted by employing the SHAKE algorithm ${ }^{46}$ to constrain bond lengths, allowing an integration time step of $2 \mathrm{fs}$. Coordinate and energy trajectories were printed out every picosecond.

All analyses were performed using the GROMOS++ software package. $^{47}$

\section{RESULTS}

LmHemQ and SaHemQ were heterologously expressed in E. coli as apoproteins (LmHemQ $\sim 30 \mathrm{mg} \mathrm{L}^{-1}$ E. coli culture; SaHemQ $\sim 50 \mathrm{mg} \mathrm{L}^{-1}$ E. coli culture) and purified as described recently. ${ }^{26}$ HemQs are likely to be present as apoproteins in their physiological resting state, because coproheme acts as both a substrate and a redox cofactor and is released after decarboxylation as protoheme IX. ${ }^{1}$ Phylogenetic analysis revealed the presence of two HemQ subfamilies, one being formed of proteins from Firmicutes and one including HemQs from Actinobacteria (Figure 1B). From the Firmicutes subfamily, the structures of the apoproteins from LmHemQ (PDB entry 4WWS) and HemQ from Geobacillus stearothermophilus (PDB entry 1T0T) have been published. In the study presented here, we focus on HemQs from Firmicutes, namely from L. monocytogenes (LmHemQ) and S. aureus (SaHemQ).

Kinetics of Binding of Coproheme and Hemin to HemQ. As a first step, the kinetics of binding of coproheme (substrate) and hemin (product) to both apoproteins was investigated by stopped-flow spectroscopy to analyze the 

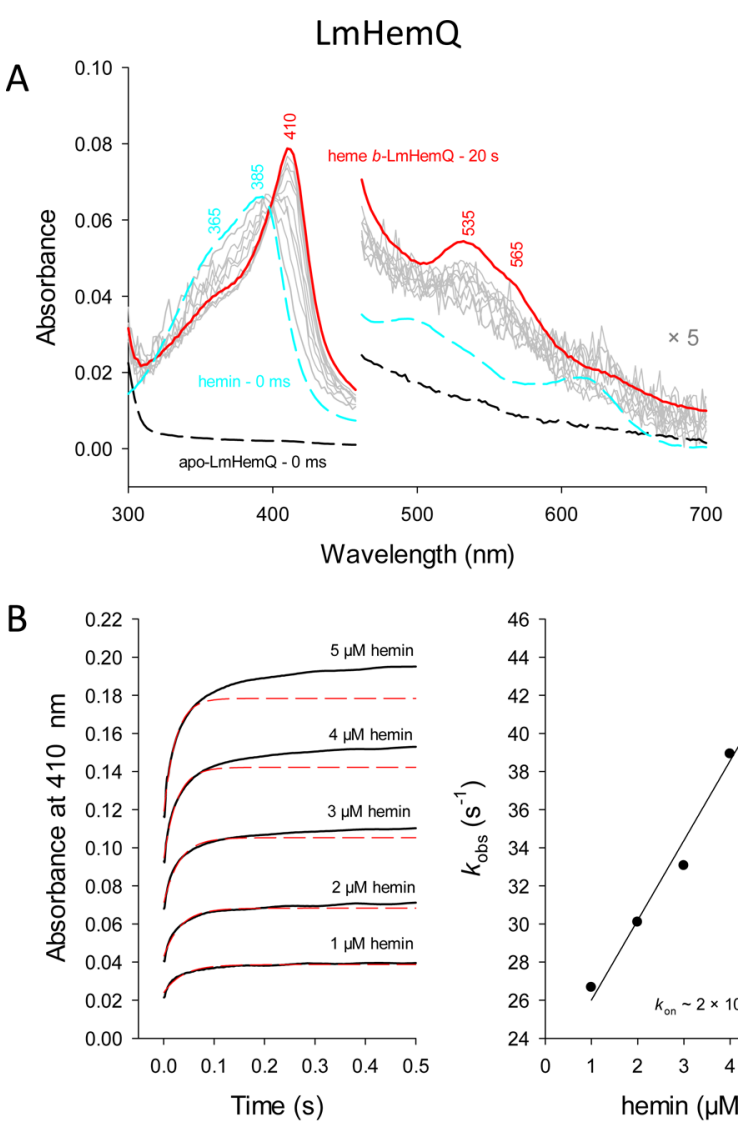

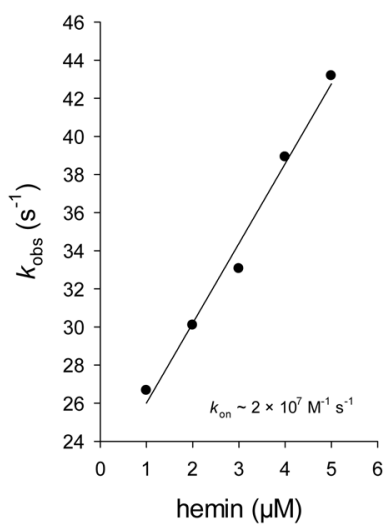

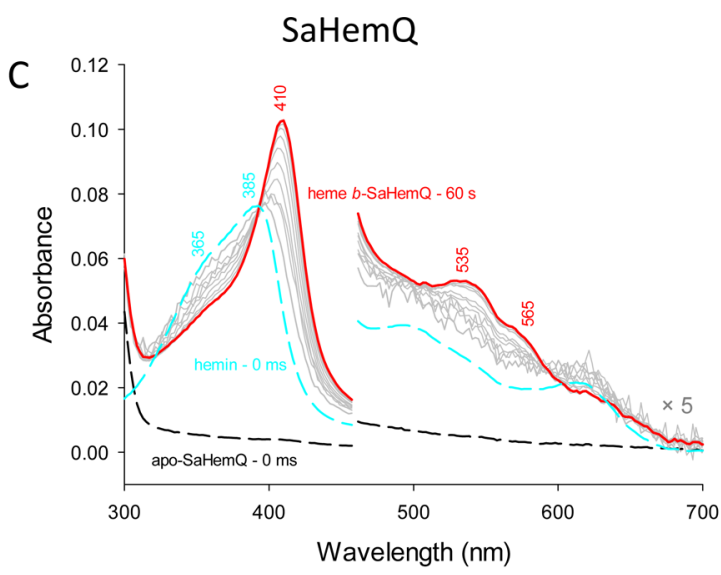

$\mathrm{D}$

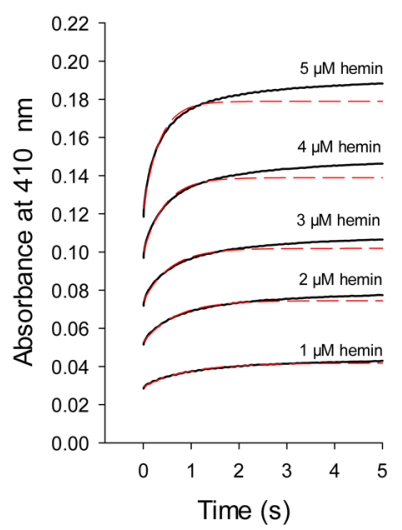

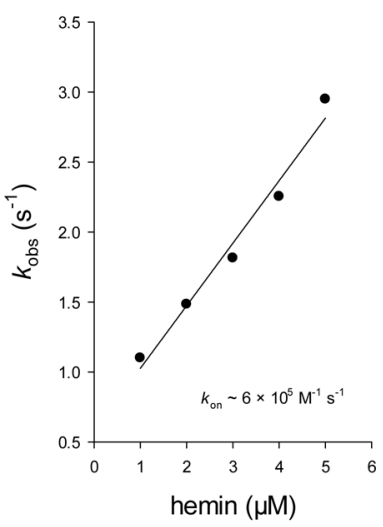

Figure 3. Kinetics of binding of hemin to apo-HemQs from L. monocytogenes and S. aureus. (A) Spectral transitions upon binding of hemin to apoLmHemQ. (C) Spectral transitions upon binding of hemin to apo-SaHemQ. (B and D) Time traces followed at $410 \mathrm{~nm}$ upon binding of hemin $(0.25-1 \mu \mathrm{M})$ to $5 \mu \mathrm{M}$ apo-LmHemQ or apo-SaHemQ: black lines for experimental time traces and red dashed lines for single-exponential fits of time traces. The right panels show plots of $k_{\mathrm{obs}}$ vs ligand concentration.

kinetics of binding and to identify intermediate spectral species. Because of (i) the high absorption of free coproheme in phosphate-buffered solutions (determined molar extinction coefficient, $\varepsilon_{390}$, of $128800 \mathrm{M}^{-1} \mathrm{~cm}^{-1}$ ) and (ii) the extremely fast binding rate (see below), it was not possible to determine binding rates when the cofactor was present in stoichiometric excess. Therefore, coproheme had to be added at subequimolar concentrations to apo-HemQ to ensure complete binding of coproheme.

Binding of coproheme occurs in a biphasic manner, with the first transition being very fast and accompanied by a decrease in absorbance at $390 \mathrm{~nm}$ [free coproheme (blue spectra in Figure $2 \mathrm{~A}, \mathrm{C})]$ and a shift of the Soret maximum to $399 \mathrm{~nm}$ (green spectra). In the second phase, the Soret absorbance peak became asymmetric, having a shoulder at $375 \mathrm{~nm}$ and a Soret maximum at $395 \mathrm{~nm}$ (red spectra in Figure 2A,C). Both LmHemQ and SaHemQ follow the same transition. Figure S1 shows the calculated spectra of all colored species in this reaction by using the Pro-K software from Applied Photophysics and the following two-step model: (i) a (coproheme) + $b$ (apo-HemQ; colorless in the Soret region) $\rightarrow c$ and (ii) $c \rightarrow$ $d$, with $c$ representing the intermediate species with a Soret maximum at $399 \mathrm{~nm}$ and $d$ representing the final spectrum of coproheme-HemQ with a Soret maximum at $395 \mathrm{~nm}\left(\varepsilon_{395}=\right.$ $68000 \mathrm{M}^{-1} \mathrm{~cm}^{-1}$ ).

The time traces at $390 \mathrm{~nm}$ depicted in panels B and D of Figure 2 show the rapid kinetics of formation of the intermediate state. Upon single-exponential fitting, $k_{\mathrm{obs}}$ values that depended on the coproheme concentration were obtained. This (although it was not possible to provide pseudo-first-order conditions) allowed us to estimate rates of binding of coproheme to apo-HemQ. Obtained $k_{\text {on }}$ rates were $\sim 1.5 \times$ $10^{8} \mathrm{M}^{-1} \mathrm{~s}^{-1}$ (LmHemQ) and $\sim 2.0 \times 10^{8} \mathrm{M}^{-1} \mathrm{~s}^{-1}$ (SaHemQ). Because of the uncertainty of $k_{\text {off }}$ values, because of the very fast $k_{\text {on }}$ values and the interplay of two consecutive reactions, it was not possible to reliably calculate $K_{\mathrm{D}}$ values from these experiments. The second phase followed as the absorbance increase at $375 \mathrm{~nm}$ was $\sim 2$ orders of magnitude slower, namely $2.2 \times 10^{6} \mathrm{M}^{-1} \mathrm{~s}^{-1}(\mathrm{LmHemQ})$ and $3.7 \times 10^{6} \mathrm{M}^{-1} \mathrm{~s}^{-1}$ (SaHemQ) (Figure 2B,D).

Binding of hemin to apo-LmHemQ or apo-SaHemQ was accompanied by spectral transitions from free hemin (Soret maximum at $385 \mathrm{~nm}$, shoulder at $365 \mathrm{~nm} ; \varepsilon_{385}=58440 \mathrm{M}^{-1}$ $\left.\mathrm{cm}^{-1}\right)^{48}$ to the corresponding heme $b$-HemQs with a Soret maximum of $410 \mathrm{~nm}\left(\varepsilon_{410}=76500 \mathrm{M}^{-1} \mathrm{~cm}^{-1}\right)$ (Figure 3A,C). Binding of hemin to both apo-HemQs was also biphasic but slower compared to coproheme binding. By following the increase in absorbance at $410 \mathrm{~nm}$, we could obtain $k_{\text {obs }}$ values of the rapid phase that depended on hemin concentration. Again because of spectral interference, it was not possible to provide a stoichiometric excess of ligand. From the plot of $k_{\mathrm{obs}}$ versus hemin concentration, $k_{\text {on }}$ and $k_{\text {off }}$ were calculated to be $2.2 \times$ $10^{7} \mathrm{M}^{-1} \mathrm{~s}^{-1}$ and $4.2 \mathrm{~s}^{-1}$ (LmHemQ) and $6.0 \times 10^{5} \mathrm{M}^{-1} \mathrm{~s}^{-1}$ and $0.4 \mathrm{~s}^{-1}$ (SaHemQ), respectively. This allowed us to calculate $K_{\mathrm{D}}$ values of 5.2 and $1.3 \mu \mathrm{M}$, respectively (Figure 3B,D). 
Because the physiological role of HemQ is to convert coproheme to heme $b$ and deliver the latter for the synthesis of heme proteins, we tested how easily heme $b$ or coproheme bound to HemQ can be extracted by apo-myoglobin (apo-Mb). Recently, apo-Mb was shown to be able to extract bound heme $b$ from $\mathrm{LmHemQ}^{26}$ and also from $\mathrm{NdCld}$ variants with a disrupted proximal H-bonding network (thus mimicking the proximal heme cavity of HemQ). ${ }^{27}$ Spectra of coproheme- and heme $b$-bound myoglobin are shown in Figure S2. Figure S3A clearly demonstrates that $10 \mu \mathrm{M}$ apo-myoglobin added to $1 \mu \mathrm{M}$ coproheme-SaHemQ (black spectrum) does not mediate the transfer of the prosthetic group. The resulting red spectrum simply reflects that of the mixture of both proteins in their initial states (Figure S3A). This indicates that coproheme is not kinetically labile during the time scale of the experiment. By contrast, mixing $1 \mu \mathrm{M}$ heme $b$-SaHemQ [black spectrum with a Soret maximum of $411 \mathrm{~nm}$ (Figure S3B)] with $10 \mu \mathrm{M}$ apomyoglobin leads to the complete transfer of the prosthetic group and the formation of $1 \mu \mathrm{M}$ myoglobin with a Soret maximum of $409 \mathrm{~nm}$.

Thermal Stability of Apo-, Heme $b$-, and CoprohemeHemQs. Because binding of a prosthetic group or cofactor and the mode of noncovalent interactions are typically reflected by the thermal stability of the respective proteins, we analyzed the thermostability of LmHemQ and SaHemQ in their apo, coproheme-bound, and heme $b$-bound states by differential scanning calorimetry. Stabilities of apo and heme $b$-bound LmHemQ were reported previously ${ }^{26}$ and determined again in this study for comparison with those of the coproheme-bound species. While temperature-mediated unfolding of apo and heme $b$-bound LmHemQ clearly follows a non-two-state transition, coproheme-LmHemQ is more stable and shows one major endothermic transition $\left(T_{\mathrm{m}}=55^{\circ} \mathrm{C}\right)$. At $>60{ }^{\circ} \mathrm{C}$, LmHemQ starts to aggregate (Figure S4).

A similar picture is seen with SaHemQ. Both apo-SaHemQ and heme $b$-SaHemQ show an almost identical unfolding behavior $\left(T_{\mathrm{m}}=59{ }^{\circ} \mathrm{C}\right)$, suggesting weak interaction of the prosthetic group with the protein. By contrast, when coproheme binds, the $T_{\mathrm{m}}$ value is increased by $14{ }^{\circ} \mathrm{C}$ (Figure 4). The difference in thermal stability between SaHemQ and LmHemQ is difficult to rationalize because of the lack of structural data for SaHemQ. Despite the high degree of similarity at the heme cavity, discrepancies in noncovalent interactions at the interfaces between the subunits of the oligomeric proteins could cause these differences as was also observed with related chlorite dismutases. ${ }^{49}$

Spectroscopic Signatures of Ferric and Ferrous Coproheme-HemQs. Next we tested the impact of $\mathrm{pH}$ on the spectral features of ferric and ferrous states of both coproheme-HemQs. UV-vis absorption and resonance Raman spectra of coproheme-SaHemQ at $\mathrm{pH} 7.4$ were reported recently. ${ }^{4}$ However, the impact of $\mathrm{pH}$ as well as spectroscopic data for other HemQs is unknown. Here we studied the spectroscopic signatures of LmHemQ and SaHemQ over a broad $\mathrm{pH}$ range using $\mathrm{UV}-$ vis absorption, circular dichroism (far-UV, near-UV, and visible), and electron paramagnetic resonance spectroscopies. UV-vis spectra of coprohemeSaHemQ were measured between $\mathrm{pH} 6.5$ and 10.0, because the protein started to precipitate below $\mathrm{pH}$ 6.5. The observed spectral signatures of the ferric protein are in agreement with previously published data, ${ }^{4}$ exhibiting a Soret maximum at 395 $\mathrm{nm}$ and a distinct shoulder at $375 \mathrm{~nm}$, the latter being verified by evaluation of the second derivative of the spectrum (Figure

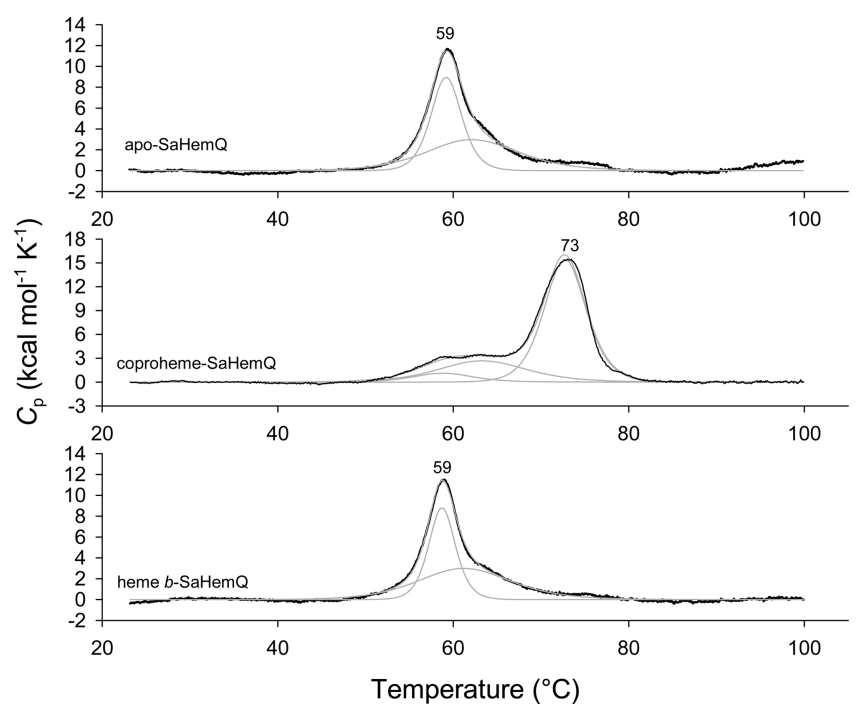

Figure 4. Thermal stability of apo-SaHemQ heme $b$-SaHemQ and coproheme-SaHemQ followed by differential scanning calorimetry. Thermograms (black lines) and fits of the endotherms (gray lines) of apo-SaHemQ coproheme-bound SaHemQ and heme $b$-SaHemQ. Conditions: $50 \mathrm{mM}$ phosphate buffer ( $\mathrm{pH} 7.0)$.

5A and Figure S5). Q-Bands are at 495 and $532 \mathrm{~nm}$, and the charge transfer band is at $630 \mathrm{~nm}$. Between $\mathrm{pH} 6.5$ and 9.0, almost identical spectra were obtained. Ferric coprohemeLmHemQ shows spectral signatures identical to those of coproheme-SaHemQ in the $\mathrm{pH}$ range of $\mathrm{pH}$ 5.0-9.0 (Figure $5 \mathrm{~A}$ and Figure S5). However, at $\mathrm{pH}$ 10.0, both proteins undergo a transition to a distinct species that has its Soret maximum still at $395 \mathrm{~nm}$ but exhibits a blue-shift of the Soret shoulder to $350 \mathrm{~nm}$ and of the CT band to $601 \mathrm{~nm}$ (LmHemQ) or $605 \mathrm{~nm}$ (SaHemQ). The spectra of ferrous coproheme-LmHemQ and coproheme-SaHemQ are very similar, having Soret maxima at $425 \mathrm{~nm}$ and a prominent band at $552 \mathrm{~nm}$ (Figure 5A, red spectra).

Spectra recorded by electron paramagnetic spectroscopy (EPR) demonstrate the existence of a predominant high-spin state of both coproheme-LmHemQ and coproheme-SaHemQ at $\mathrm{pH} 7.0$ (Figure 5B). With both proteins, simulation of the spectra (Table 1 ) suggests the presence of a dominating slightly rhombic axial high-spin species together with some low-spin form. Very similar high-spin spectra were seen between $\mathrm{pH} 6.0$ and 9.0, whereas at $\mathrm{pH}$ 10.0, a pure low-spin form was present (data not shown). Upon addition of $1 \mathrm{mM}$ cyanide, both proteins were completely converted to the corresponding lowspin complexes (insets of Figure 5B).

Electronic circular dichroism (ECD) spectroscopy of apoHemQ coproheme-HemQ and heme $b$-HemQ suggests similar secondary structure compositions. The minima at 209 and $221 \mathrm{~nm}$ (Figure S6) reflect the presence of $\alpha$-helices and $\beta$ sheets as predicted by examination of the X-ray structures of apo-LmHemQ (PDB entry 4WWS) and HemQ from G. stearothermophilus (PDB entry 1TOT). The observed differences are small, e.g., reflected by ratio of ellipticities at 220 and $209 \mathrm{~nm}$, but followed the same trend in LmHemQ and SaHemQ. Importantly, the spectrum of heme $b$-SaHemQ (which is highly similar to that of heme $b$-LmHemQ) is significantly different from that previously reported, where the apoprotein exhibits a minimum at $227 \mathrm{~nm}$ and the heme $b$ - 

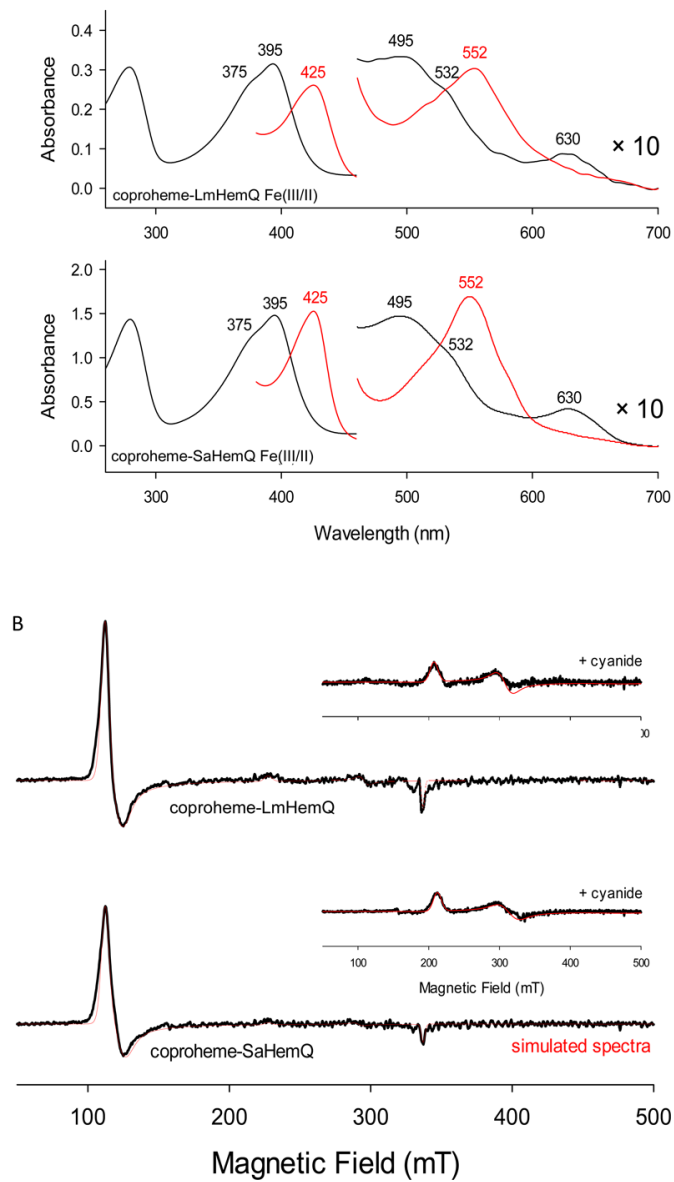

Figure 5. Spectral signatures of ferric and ferrous coproheme-HemQs. (A) UV-vis absorption spectra of LmHemQ and SaHemQ in their ferric (black line) and ferrous (red line) states. Conditions: $50 \mathrm{mM}$ phosphate buffer ( $\mathrm{pH} 7.0$ ). (B) EPR spectra of $50 \mu \mathrm{M}$ coprohemeLmHemQ and coproheme-SaHemQ. Experimental traces are shown as solid black lines, and simulated spectra (using EasySpin software) are shown as red lines. The inset depicts EPR spectra recorded in the presence of $1 \mathrm{mM}$ cyanide. Conditions: $10 \mathrm{~K}$ and $200 \mathrm{mM}$ phosphate buffer ( $\mathrm{pH} 7.0)$.

bound form at $234 \mathrm{~nm}$, which is unusual and might indicate misfolding. ${ }^{25,50}$

ECD spectroscopy in the near-UV and visible region revealed interesting distinct spectral features of coproheme-HemQ and heme $b$-HemQ. Free coproheme is an achiral compound but upon binding to apo-HemQ becomes chiral. Both coprohemeHemQs exhibited a minimum in ellipticity at $388 \mathrm{~nm}$ (Figure S6). By contrast, heme $b$-HemQs showed a maximum in ellipticity at 414-416 $\mathrm{nm}$. The cyanide complexes of both coproheme-HemQs have distinct maxima at 328 and $385 \mathrm{~nm}$ and minima at 360 and $421 \mathrm{~nm}$, respectively. The insets of Figure S6 show the corresponding UV-vis spectra for comparison.

Ligand Binding. The binding of cyanide to heme proteins is often used to probe the accessibility of the ligand to the active site as well as the architecture of the distal heme cavity. As demonstrated by spectroscopic means, cyanide is able to enter the active site of copropheme-HemQs and bind to the iron. To probe the kinetics and thermodynamics of this process, $k_{\mathrm{on}}, k_{\mathrm{off}}$, and $K_{\mathrm{D}}\left(=k_{\text {off }} / k_{\text {on }}\right)$ were determined by stopped-flow spectroscopy. Upon addition of cyanide, both HemQs were directly converted to their low-spin complex $(S=1 / 2)$, exhibiting a redshifted Soret maximum at $410 \mathrm{~nm}$ (LmHemQ) and $411 \mathrm{~nm}$ (SaHemQ) (isosbestic point of transition at $402 \mathrm{~nm}$ ) (Figure $6 \mathrm{~A}, \mathrm{D})$. With both proteins, ligand binding followed at $410 \mathrm{~nm}$ was monophasic and $k_{\mathrm{obs}}$ values could be obtained from singleexponential fits (Figure 6B,E). The apparent second-order rate constants for cyanide binding $\left(k_{\text {on }}\right)$ at $\mathrm{pH} 7.0$ were calculated from the slope of the linear plots of $k_{\text {obs }}$ versus cyanide concentration (Figure 6C,F), whereas the dissociation rate constant $\left(k_{\text {off }}\right)$ was determined from the intercepts of those linear plots. Both HemQs exhibited similar kinetics and thermodynamics of cyanide binding with a $k_{\text {on }}$ of $1.0 \times 10^{4}$ $\mathrm{M}^{-1} \mathrm{~s}^{-1}$, a $k_{\text {off }}$ of $0.17 \mathrm{~s}^{-1}$, and a $K_{\mathrm{D}}$ (dissociation constant) of $16.4 \mu \mathrm{M}(\mathrm{LmHemQ})$ and a $k_{\text {on }}$ of $9.2 \times 10^{3} \mathrm{M}^{-1} \mathrm{~s}^{-1}$, a $k_{\text {off }}$ of $0.09 \mathrm{~s}^{-1}$, and a $K_{\mathrm{D}}$ of $9.9 \mu \mathrm{M}$ (SaHemQ). Binding of cyanide to coproheme-LmHemQ and coproheme-SaHemQ did not depend on $\mathrm{pH}$ (data not shown), indicating that there is no proton acceptor involved in cyanide (i.e., HCN) binding close to the active site. Around $\mathrm{pH} 9$, there was a slight increase in

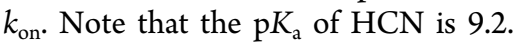

Spectroelectrochemistry. Next we performed temperature-controlled spectroelectrochemical redox titrations between 10 and $25{ }^{\circ} \mathrm{C}$ to analyze the thermodynamics of the reduction of ferric coproheme-HemQ to ferrous coprohemeHemQ. At $20^{\circ} \mathrm{C}$, the standard reduction potentials, $E^{\circ \prime}$, of the $\mathrm{Fe}(\mathrm{III}) / \mathrm{Fe}$ (II) couple of coproheme-LmHemQ and coproheme-SaHemQ were determined to be $205 \pm 3$ and $207 \pm 3$ $\mathrm{mV}$, respectively, versus the SHE. The redox reactions showed a clear isosbestic point at $405 \mathrm{~nm}$ (Figure 7A).

To study the mechanism of $E^{\circ \prime}$ modulation, we investigated its temperature dependence. This allows parametrization of the corresponding enthalpic $\left(\Delta H^{\circ \prime}{ }_{\text {rc }}\right)$ and entropic $\left(\Delta S^{\circ \prime}{ }_{\text {rc }}\right)$ contributions of the $\mathrm{Fe}(\mathrm{III})$ to $\mathrm{Fe}(\mathrm{II})$ reduction reaction. ${ }^{37}$ Reduction of coproheme-SaHemQ from $\mathrm{Fe}(\mathrm{III})$ to $\mathrm{Fe}(\mathrm{II})$ is enthalpically favorable $\left(\Delta H^{\circ}{ }_{r c}=-67 \pm 4 \mathrm{~kJ} \mathrm{~mol}^{-1}\right)$ but entropically disfavored $\left(\Delta S^{\circ \prime}{ }_{\mathrm{rc}}=-296 \pm 14 \mathrm{~J} \mathrm{~mol}^{-1} \mathrm{~K}^{-1}\right)$ (Figure $7 \mathrm{~B}$ and Table 2). As a consequence, the resulting

Table 1. EPR Parameters of HemQs at $\mathrm{pH} 7.0$

\begin{tabular}{|c|c|c|c|c|c|c|c|c|c|}
\hline \multirow[b]{2}{*}{ protein } & \multirow[b]{2}{*}{ species } & \multirow[b]{2}{*}{$g_{1}$} & \multirow[b]{2}{*}{$g_{2}$} & \multirow[b]{2}{*}{$g_{3}$} & \multirow[b]{2}{*}{$R^{a}(\%)$} & \multirow[b]{2}{*}{$I(\%)$} & \multicolumn{3}{|c|}{$g$ strain } \\
\hline & & & & & & & $g_{1}$ & $g_{2}$ & $g_{3}$ \\
\hline \multirow[t]{2}{*}{ SaHemQ } & HS & 5.95 & 5.55 & 1.99 & 2.5 & 87 & 0.30 & 0.60 & 0.02 \\
\hline & LS & 2.95 & 2.30 & $\leq 1$ & & 13 & 0.07 & 0.05 & 0.08 \\
\hline \multirow[t]{2}{*}{ LmHemQ } & HS & 5.95 & 5.63 & 1.99 & 2.0 & 87 & 0.30 & 0.60 & 0.01 \\
\hline & LS & 2.95 & 2.27 & $\leq 1$ & & 13 & 0.08 & 0.05 & 0.08 \\
\hline SaHemQ-CN & LS & 3.16 & 2.13 & $\leq 1$ & & 100 & 0.20 & 0.20 & 0.20 \\
\hline LmHemQ-CN & LS & 3.22 & 2.17 & $\leq 1$ & & 100 & 0.20 & 0.15 & 0.20 \\
\hline
\end{tabular}

${ }^{a} R$ is the rhombicity of HS signals calculated according to the method of Peisach et al. ${ }^{34}(\Delta g / 16)$. 

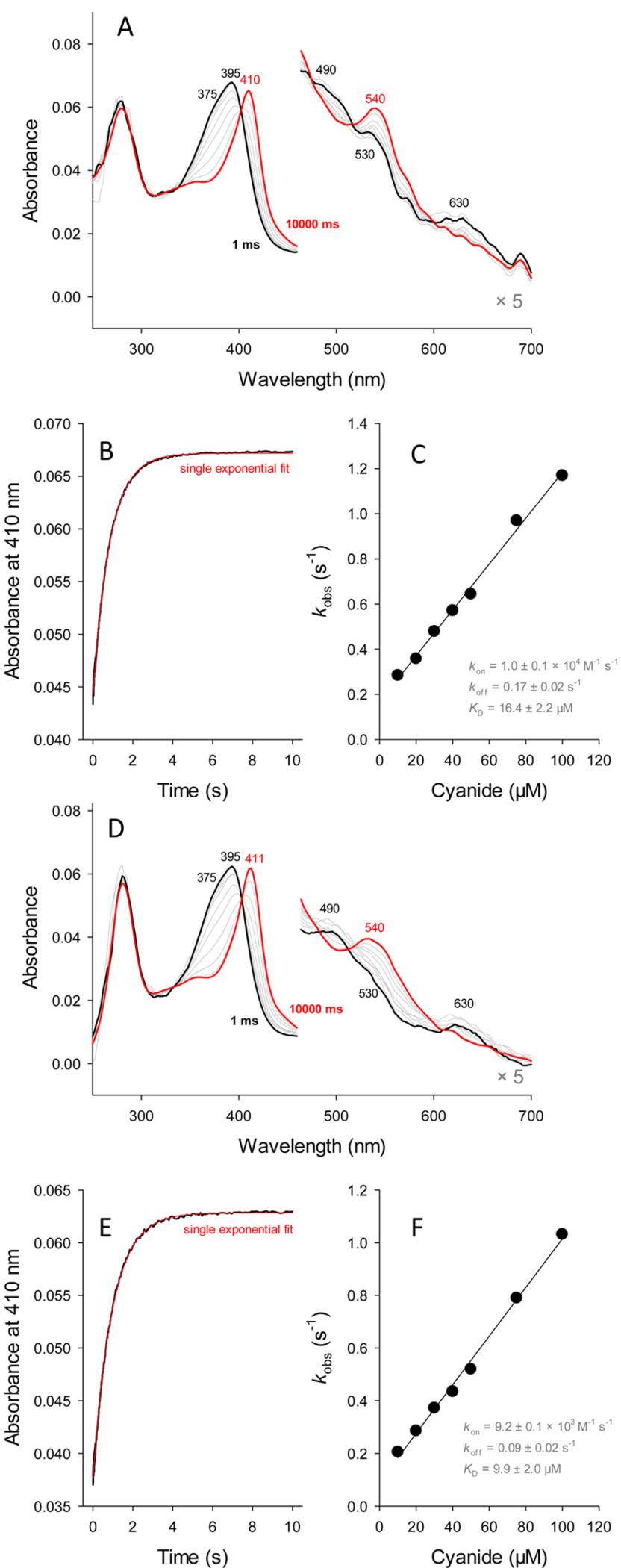

Figure 6. Kinetics and thermodynamics of binding of cyanide to coproheme-HemQs. Spectral changes upon reaction of $1 \mu \mathrm{M}$ ferric coproheme-LmHemQ (A) and coproheme-SaHemQ (D) with 100 $\mu \mathrm{M}$ cyanide measured in the conventional stopped-flow mode. Typical time traces at $410 \mathrm{~nm}$ with a single-exponential fit for LmHemQ (B) and SaHemQ (E). Linear dependence of $k_{\mathrm{obs}}$ values from the cyanide concentration for LmHemQ (C) and SaHemQ (F). Conditions: 50 $\mathrm{mM}$ phosphate buffer ( $\mathrm{pH}$ 7.0).
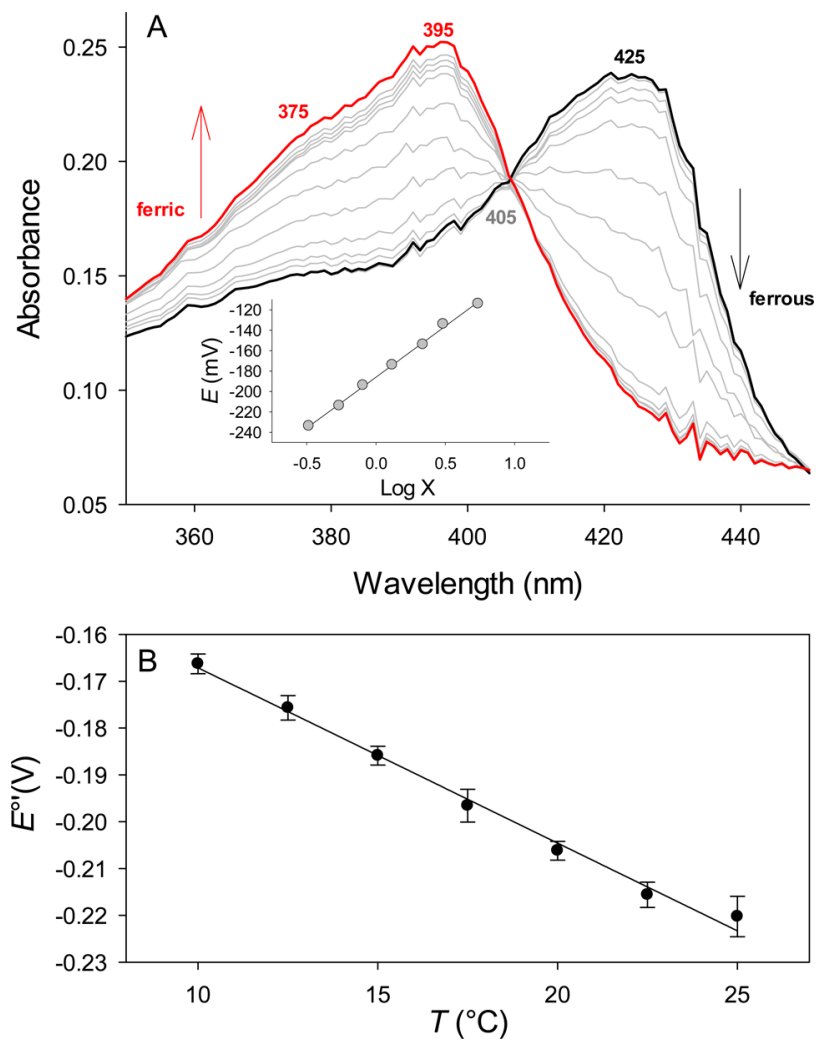

Figure 7. Spectroelectrochemistry and redox thermodynamics of coproheme-SaHemQ. (A) Data for ferrous SaHemQ (at $-414 \mathrm{mV}$ vs the SHE) are colored black and data for ferric SaHemQ (at $-34 \mathrm{mV}$ vs the SHE) red. Spectra recorded at various potentials between -414 and $-34 \mathrm{mV}$ vs the SHE are colored gray. The Nernst plot is shown as an inset, where $\log X$ is $\log \left[\left(A^{\circ}{ }_{425}-A_{425}\right) /\left(A^{\circ}{ }_{395}-A_{395}\right)\right]$. (B) Redox thermodynamics of $\mathrm{SaHemQ}_{i}$ temperature dependence of the reduction potential for SaHemQ. The slope of the plot yields $\Delta S^{\circ \prime}$ / F. Solid lines are least-squares fits to the data points.

enthalpic contribution to $E^{\circ \prime}$ at $25{ }^{\circ} \mathrm{C}\left(-\Delta H^{\circ \prime}{ }_{\text {rc }} / F=694 \pm 43\right.$ $\mathrm{mV})$ partially compensates for the entropic stabilization of the ferric state $\left(T \Delta S^{\prime \prime}{ }_{\mathrm{rc}} / F=-915 \pm 41 \mathrm{mV}\right)$. Table 2 compares these thermodynamic data with those of homologous pentameric $(\mathrm{NdCld})$ and dimeric $(\mathrm{NwCld})$ chlorite dismutases. $^{51}$

Molecular Dynamics Simulation of CoprohemeLmHemQ. To rationalize the biochemical and biophysical observations described above, we performed $\mathrm{MD}$ simulations of apo-HemQ heme $b$-HemQ and coproheme-HemQ. Coproheme was placed in silico at the predicted heme binding site of all five subunits of apo-LmHemQ (PDB entry 4WWS). ${ }^{26}$ In the starting position, H174 acted as the proximal ligand and the propionates at positions 6 and 7 were positioned as in the heme $b$ enzymes NdCld and NwCld. During the $30 \mathrm{~ns}$ simulation, the overall structure remained intact (Figure 8A). In agreement with the DSC experiments, the apo structure seems less stable than the coproheme-HemQ. This is most pronounced for simulations of the isolated monomers, which start to unfold at residues $150-175$ in the absence of a substrate, while the coproheme-HemQ monomer remains as stable as the corresponding chain in the pentameric structures (Figure S8). The greatest structural variability was seen in the flexible loop (V107-K141) (Figure 8F) at the entrance of the substrate channel and in the $\alpha$-helix (M164-Y181) on the proximal heme side that harbors H174 (Figure 8G and Figure S7). H174 
Table 2. Standard Reduction Potentials (vs SHE) of Coproheme-HemQs and Chlorite Dismutases

\begin{tabular}{|c|c|c|c|c|c|c|c|c|}
\hline & $E^{\circ \prime}(\mathrm{mV})$ & $\begin{array}{c}\Delta S^{\circ \prime} \\
\left(\mathrm{J} \mathrm{mol}^{-1} \mathrm{~K}^{-1}\right)\end{array}$ & $\underset{\left(\mathrm{kJ} \mathrm{mol}^{-1}\right)}{\Delta H^{\circ}{ }^{\mathrm{rc}}}$ & $\left(\begin{array}{c}\Delta S^{\mathrm{ol}} \\
(\mathrm{mol} \\
-1 \\
\mathrm{~K}^{-1}\end{array}\right)$ & $\begin{array}{c}-\Delta H_{\mathrm{rc}}^{\circ \prime} / F \\
(\mathrm{mV})\end{array}$ & $\begin{array}{l}T \Delta S_{\mathrm{rc}}^{\mathrm{or}} / F \\
\quad(\mathrm{mV})\end{array}$ & $\begin{array}{c}-F E^{\circ \prime}\left[=\Delta H^{\circ \prime}{ }^{\mathrm{rc}(\mathrm{int})}\right] \\
(\mathrm{kJ} \mathrm{mol})^{-1}\end{array}$ & ref \\
\hline SaHemQ & $-207 \pm 3$ & $-361 \pm 14$ & $-67 \pm 4$ & $-296 \pm 14$ & $694 \pm 43$ & $-915 \pm 41$ & $21.3 \pm 0.4$ & this study \\
\hline LmHemQ & $-205 \pm 3$ & $\mathrm{ND}^{a}$ & $\mathrm{ND}^{a}$ & $\mathrm{ND}^{a}$ & $\mathrm{ND}^{a}$ & $\mathrm{ND}^{a}$ & $\mathrm{ND}^{a}$ & this study \\
\hline NdCld & $-113 \pm 5$ & & $29 \pm 6$ & $63 \pm 20$ & $-305 \pm 60$ & $194 \pm 60$ & $10.9 \pm 0.1$ & 50 \\
\hline NwCld & $-119 \pm 5$ & & $40 \pm 4$ & $95 \pm 13$ & $-413 \pm 40$ & $292 \pm 40$ & $11.5 \pm 0.1$ & 50 \\
\hline
\end{tabular}
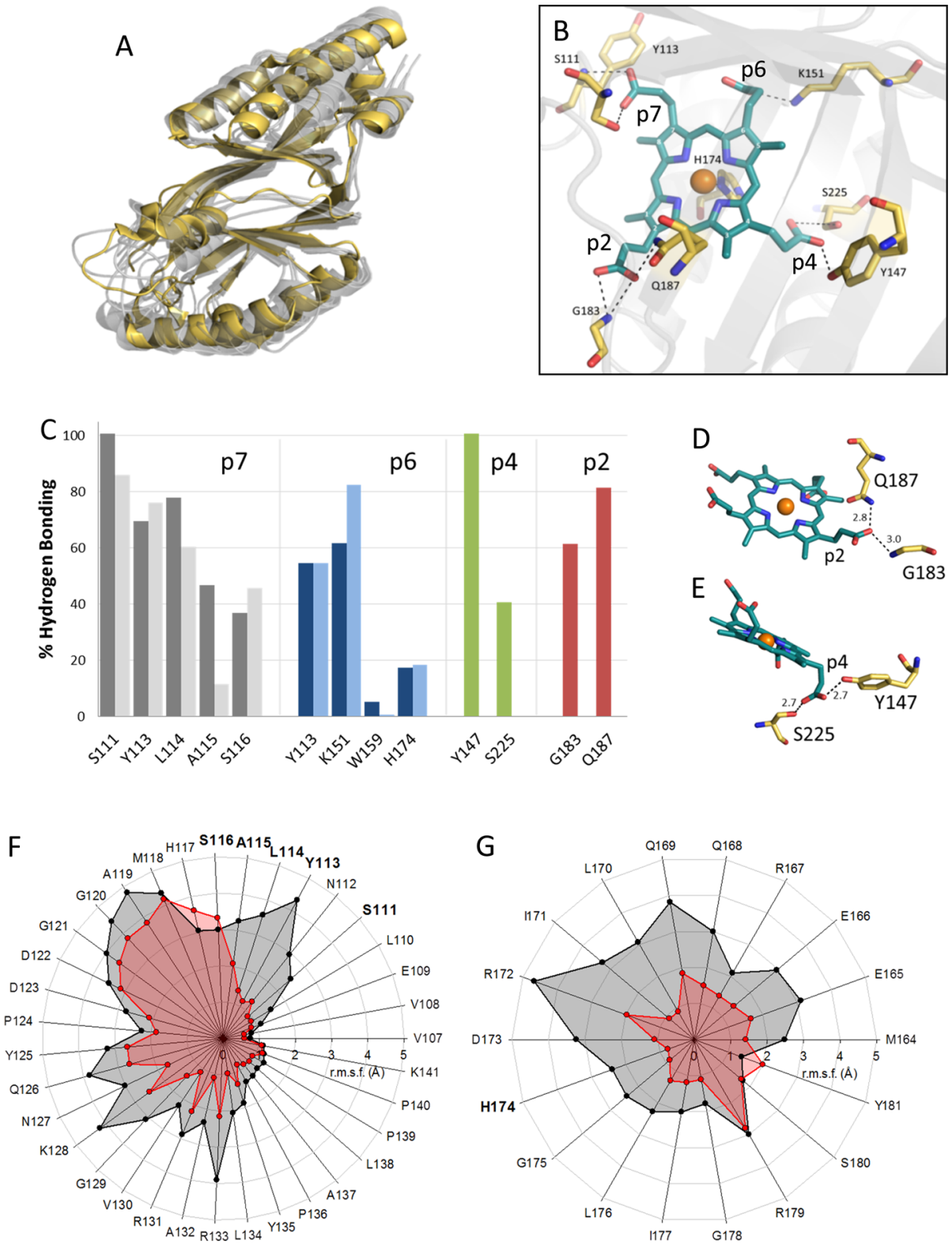

Figure 8. Proposed interactions of coproheme with HemQ based on MD simulations. (A) Overlay of subunit structures of apo-LmHemQ (yellow, PDB entry 4WWS) and coproheme-LmHemQ (gray, chains A-E) from MD simulations (last frames). (B) Hydrogen bonding network of coproheme in LmHemQ (last frame, chain A), in which positions of porphyrin substituents are numbered in white. (C) H-Bonding interaction of atoms of respective amino acids and coproheme propionates (for details, see Table S1) and for heme $b$ propionates. $\mathrm{H}$-Bonds formed (in percent) throughout the simulation time as averages over all five subunits are represented as dark (coproheme) and light (heme $b$ ) gray bars (residues $\mathrm{H}$ bonded to propionate at position 7), dark (coproheme) and light (heme $b$ ) blue bars (p6), red bars (p4), and green bars (p2). H-Bonding of (D) p2 to coproheme (last frame, chain A) and (E) p4 to coproheme (last frame, chain C). (F) Root-mean-square fluctuations (in angstroms) for all atoms (averages over all five chains) of the flexible loop on the entrance to the coproheme/heme $b$ binding site between residues V107 and K141 in apoLmHemQ (black) and coproheme-LmHemQ (red) over 30 ns simulations. (G) Root-mean-square fluctuation analysis as in panel F for the $\alpha$-helix (M164-Y181) that harbors the proximal histidine (H174). 
coordinates the coproheme for the vast majority of the time, while a water molecule serves as a sixth ligand, as expected for a high-spin complex. Interestingly, R179 of the $\alpha$-helix (M164Y181) shows the same structural variability in the simulation of apo-LmHemQ and coproheme-LmHemQ in contrast to other residues in this helix (Figure 8G). In apo-LmHemQ R179 is blocking the potential coproheme/substrate binding site, whereas in coproheme-LmHemQ (after a $30 \mathrm{~ns}$ simulation), R179 adopts a completely different conformation; therefore, the coproheme/substrate binding site is highly accessible (Figure S9).

The MD data show that all four propionates interact with the protein (Figure $8 \mathrm{~B}, \mathrm{C}$ ). The most prominent $\mathrm{H}$-bonding partners for the propionates at positions 6 (p6) and $7(\mathrm{p} 7)$, which are not modified during the enzymatic reaction, are the side chains of Y113 and K151 for p6 and the side chain of S111 and the backbone of $\mathrm{Y} 113$ for $\mathrm{p} 7$. Those propionates that are decarboxylated, namely, p2 and p4, interact with G183 and Q187 as well as Y147 and S225, respectively. Analysis of $\mathrm{H}$ bond formation (average over all five subunit chains) over the entire simulation time is depicted in Figure 8C, whereas Table S1 shows all hydrogen bonds throughout the 30 ns simulation over all five subunits.

The propionates $\mathrm{p} 2$ and $\mathrm{p} 4$ that are cleaved off during catalysis are thus of significant interest. Molecular dynamics simulation suggests that the propionate at position 2 can form H-bonds for more than $5 \%$ of the simulation time, with only G183 and Q187 (Figure 8D), whereas p4 builds significant Hbonds with Y147 and S225, respectively (Figure 8E and Table S1). The most prominent noncovalent interaction is formed between the $\mathrm{O} 1$ or $\mathrm{O} 2$ atom of $\mathrm{p} 4$ and the oxygen of Y147 ( 100\%), whereas the S225 side chain shows H-bonding to p4 for 41\%; it must be mentioned that in SaHemQ S225 (in LmHemQ) aligns with T223 (Figure 1A). Therefore, we can conclude that Y147 is the primary and most important $\mathrm{H}$ bonding partner of $\mathrm{p} 4$.

Propionate at position $2(\mathrm{p} 2)$ is the closest to the protein surface and partly solvent-exposed. It can form $\mathrm{H}$-bonds with the G183 main chain and Q187 side chain. The strongest Hbond connections are formed by Q187 (81\%) and by the backbone $\mathrm{N}$ atom of G183 (61\%) (Table S1).

Other data derived from MD simulations of heme $b$-HemQ show that $\mathrm{p} 6$ and $\mathrm{p} 7$ interact with the same amino acid residues as in coproheme-HemQ (Figure $8 \mathrm{C}$ ). The significance of amino acid residues in coordinating $\mathrm{p} 6$ and $\mathrm{p} 7$ of heme $b$ and coproheme is similar; only in heme $b$-HemQ is the interaction of A115 with p7 less important, whereas the H-bond between $\mathrm{K} 151$ and $\mathrm{p} 6$ is even more dominant.

\section{DISCUSSION}

Following the availability of large numbers of microbial genomes and comprehensive evaluation of heme biosynthesis pathways together with biochemical analysis, it became clear that chlorite dismutase-like proteins in Firmicutes and Actinobacteria are essential for heme biosynthesis. ${ }^{1,2}$ These proteins, finally named HemQs, ${ }^{24}$ were proposed to oxidatively decarboxylate coproheme (formed by $\mathrm{HemH}$, which inserts ferrous iron into coproporphyrin) into protoheme (heme $b$ ). ${ }^{1}$ This conversion was shown in vitro to follow two consecutive decarboxylation steps that were shown to depend on an excess of hydrogen peroxide or peracetic acid. ${ }^{4}$ In vitro, the reaction occurs with the conversion of $\mathrm{p} 2$ to the vinyl group at pyrrole ring A forming the monovinyl, monopropionate deuteroheme isomer as an intermediate (incorrectly named three-propionate harderoheme isomer III in the literature), which can be released from HemQ. In a slower reaction, the latter is decarboxylated, thereby converting $\mathrm{p} 4$ to the vinyl substituent at pyrrole ring B. ${ }^{4}$ It is not known whether these reactions occur in vivo.

So far, there are no Actinobacterial HemQ structures in the PDB, but four homopentameric structures of (initially annotated as chlorite dismutases) HemQs from Firmicutes and Archaea do exist, namely from G. stearothermophilus (1TOT), Thermoplasma acidophilum (3DTZ), Thermus thermophilus (1VDH), and L. monocytogenes (4WWS). None of those structures possess bound heme or coproheme. As a consequence, the position of the substrate coproheme and the product protoheme can be presumed only by analogy to known Clds. It is not known if coproheme binding and/or heme $b$ release involves conformational changes or structural rearrangements like in ferrochelatase. ${ }^{52}$ This lack of knowledge motivated us to study the mode of binding and interactions of coproheme and heme $b$ with the HemQ from two troublesome pathogens, namely, S. aureus (SaHemQ) and L. monocytogenes (LmHemQ).

We showed that both apo-SaHemQ and apo-LmHemQ behave identically and bind coproheme very rapidly in a biphasic reaction with (i) a spectrally clearly visible intermediate with a Soret maximum at $399 \mathrm{~nm}$ and (ii) kinetically well separated phases $\left(\sim 1.5-2 \times 10^{8} \mathrm{M}^{-1} \mathrm{~s}^{-1}\right.$ vs 2.2-3.3 $\left.\times 10^{6} \mathrm{M}^{-1} \mathrm{~s}^{-1}\right)$. Because of the high binding rate and the spectral interference with the ligand coproheme, it was not possible to determine an exact bimolecular rate constant for the fast phase. However, the findings clearly demonstrate that (i) apo-HemQ efficiently binds coproheme even in the presence of a subequimolar or equimolar ligand concentration and (ii) this suggests that coproheme released by $\mathrm{HemH}$ will not accumulate in vivo.

Binding of coproheme to apo-HemQ includes coordination by the proximal histidine (H174) and establishment of many noncovalent interactions, especially between the four propionate side chains and the protein. The far-UV ECD spectra suggest only very small but evident differences in the overall secondary structure composition of apo-HemQ and coproheme-HemQ, which is indeed observed in the MD simulations. Apo-HemQ shows $34.0 \% \quad \alpha$-helical and $26.2 \% \quad \beta$-sheet conformations, averaged over the simulation and the five chains, while for coproheme-HemQ, these values are 33.6 and $26.3 \%$, respectively, according to the DSSP classification. ${ }^{53}$ However, the MD simulations clearly demonstrate structural variability in regions that are involved in ligand accessibility and binding. HemQs of Firmicutes (in contrast to Actinobacterial counterparts) have an additional loop at the mouth of the (putative) active site (V107-K141 in LmHemQ) (Figure 8F). The $\alpha$-helix (M164-Y181) that harbors H174 exhibits significantly increased flexibility in apo-HemQ compared to that in coproheme-HemQ and seems to have a mobile arginine (R179), which possibly has a gating function (Figure 8G and Figure S9). In contrast to the case for Clds, this proximal histidine is not part of an extended H-bonding network that decreases the flexibility of the corresponding helix in Clds. The biphasic behavior of binding of coproheme (but also hemin) to apo-HemQ could be explained by initial rapid binding of the prosthetic group by $\mathrm{H} 174$ followed by a slower rearrangement of this $\alpha$-helix and establishment of noncovalent interactions between the prosthetic group and the protein. 
The UV-vis spectrum of ferric coproheme-HemQ exhibits an asymmetric Soret band with a maximum at $395 \mathrm{~nm}$ and a prominent shoulder at $375 \mathrm{~nm}$. The CT band at $630 \mathrm{~nm}$ together with the EPR spectra clearly suggests the presence of predominating high-spin heme between $\mathrm{pH} 5$ and 9. At higher $\mathrm{pH}$, low-spin coproheme-HemQ dominates, most probably reflecting the alkaline transition and the presence of an $\mathrm{OH}^{-}$ ligand at the sixth coordination site. The origin of the asymmetric Soret peak was hypothesized to be partial conversion of coproheme to monovinyl, monopropionate deuteroheme, ${ }^{4}$ but this reaction would need hydrogen peroxide (which was not present in our experiments) and as a consequence should lead to heterogeneity of the active site structure, which should be reflected by the kinetics of ligand binding. However, the latter was monophasic and very similar to both proteins $\left(k_{\text {on }}=0.9-1.0 \times 10^{4} \mathrm{M}^{-1} \mathrm{~s}^{-1} ; K_{\mathrm{D}}=9.9-16.4\right.$ $\mu \mathrm{M})$. On the basis of (i) the symmetric spectrum of the intermediate formed during coproheme binding and (ii) the heme $b$-HemQ spectra that also lost the shoulder at $375 \mathrm{~nm}$, it is reasonable to assume that the asymmetry of the Soret band in coproheme-HemQ is related to specific interactions of $\mathrm{p} 2$ and p4 with the protein that are not yet established in the intermediate species and already absent in heme $b$-HemQ.

Compared to those of homologous Clds (with heme $b$ at the active site), the cyanide binding rate in coproheme-HemQ is $\sim 2$ orders of magnitude slower, suggesting a more restricted accessibility of the ligand to the heme cavity. This would also hold for the putative substrate hydrogen peroxide. Additionally, the calculated $K_{\mathrm{D}}$ values of cyanide complexes in Clds are 3-5 times smaller than those of coproheme-HemQ. ${ }^{51}$ In structures of cyanide-Cld complexes, the conserved distal arginine binds to ligands like cyanide, azide, and thiocyanate. ${ }^{13,14,54}$ In HemQs from Firmicutes, this arginine is missing and exchanged with a glutamine, which, because of the chemical nature of its side chain, has a lower capacity to electrostatically attract and bind the negatively charged ions.

Recently, we have demonstrated that both lineages of homologous and heme $b$-carrying Clds that differ in oligomeric state and subunit architecture exhibit an almost identical standard reduction potential $\left(E^{\circ \prime}\right)$ of the $\mathrm{Fe}(\mathrm{III}) / \mathrm{Fe}$ (II) couple of -113 to $-119 \mathrm{mV}$ at $\mathrm{pH} 7.0 .^{51}$ This compares with values of -205 and $-207 \mathrm{mV}$ versus the SHE for coproheme-LmHemQ and coproheme-SaHemQ, respectively. This clearly suggests that the native stable oxidation state of coproheme-HemQ is $\mathrm{Fe}(\mathrm{III})$, which is important for reaction with hydrogen peroxide. The question of how $\mathrm{Fe}(\mathrm{II})$-coproheme delivered by $\mathrm{HemH}^{1,2}$ is oxidized to $\mathrm{Fe}$ (III)-coproheme before incorporation into HemQ remains.

Besides the more negative $E^{\circ \prime}$ value, the mechanism of modulation of $E^{\circ \prime}$ by temperature was completely different in coproheme-HemQ and Clds. In the latter, reduction of the ferric protein to the ferrous state was shown to be entropically favored but enthalpically disfavored (Table 2), whereas the transition of ferric coproheme-SaHemQ to ferrous coprohemeSaHemQ is enthalpically favored but entropically disfavored. Reduction-induced changes in entropy $\left(\Delta S^{\circ \prime}{ }_{\text {rc }}\right)$ reflect changes in the conformational degree of freedom of the polypeptide chain(s) and solvent reorganization effects. Negative $\Delta S^{\circ \prime}{ }_{\text {rc }}$ values are often associated with proteins undergoing redoxinduced conformational changes, such as adrenodoxin. ${ }^{55}$ When reduction-induced three-dimensional structural changes are small $\left(\Delta S^{\circ}{ }_{\text {rcint }} \approx 0\right)$, as observed for many heme proteins, the change in entropy would mainly reflect solvent reorganization
$\left(\Delta S^{\circ}{ }_{\text {rcssolv }}\right){ }^{56-59}$ In these proteins, negative $\Delta S^{\circ \prime}{ }_{\text {rc,solv }}$ values indicate a reduction-induced increase in the level of solvent ordering, in particular within the heme cavity, and are generally observed in systems containing buried metal sites, characterized by a limited solvent accessibility. ${ }^{56-59}$ Therefore, the obtained negative values of $\Delta S^{\circ{ }^{\prime}{ }_{\text {rc }}}\left(\approx \Delta S^{\circ \prime}{ }_{\text {rc,solv }}\right)$ in coproheme-SaHemQ might suggest limited solvent accessibility and mobility at the heme cavity. Limited accessibility to the distal heme cavity is also reflected by the significantly slower kinetics of cyanide binding in coproheme-HemQ compared to Clds.

An important contribution to the difference existing between

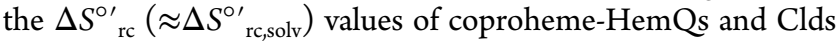
could derive from the two additional carboxylate groups of coproheme, which change the electrostatic charge of the corresponding fully deprotonated ferrous and ferric forms to -4 and -3 , respectively. The negative $\Delta S^{\circ \prime}$ rc,solv values of coproheme-HemQ are consistent with the enhanced electrostatic interaction between the ferrous coproheme (overall charge of -4) and the water molecules in the distal cavity as compared with that of the ferric form (overall charge of -3 ), leading to an increased level of solvent ordering upon reduction. ${ }^{56-59}$ Moreover, the two additional carboxylates in coproheme-HemQ could strengthen the H-bond network compared to that of Cld, significantly altering the reductioninduced solvent reorganization within the heme cavity.

Reduction-induced solvent reorganization effects usually induce compensatory enthalpy and entropy changes. The corresponding enthalpic contribution can be factored out from the measured enthalpy change ${ }^{56-59}$ and allows estimation of the protein-based contribution $\left(\Delta H^{\circ \prime}{ }_{\text {rc,int }}\right)$ to $\Delta G^{\circ{ }^{\circ}}{ }^{\prime}=-n F E^{\circ \prime}$ $=\Delta H^{\circ}{ }_{r c}-T \Delta S^{\circ}{ }_{r c}=\Delta H^{\circ \prime}{ }_{r c, i n t}+\Delta H^{\circ \prime}{ }_{r c, s o l v}-T \Delta S^{\circ \prime}{ }_{r c, s o l v}$. Solvent reorganization effects typically cancel in enthalpy and entropy; therefore, it follows that $\Delta G^{\circ{ }^{\prime}}{ }_{\mathrm{rc}}=-n F E^{\circ \prime}=\Delta H^{\circ{ }^{\prime}}{ }_{\mathrm{rc}, \text { int }}$

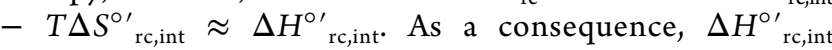
corresponds to $23.3 \mathrm{~kJ} / \mathrm{mol}$, which is more positive compared to that of Clds (Table 2). Primarily, $\Delta H^{\circ \prime}{ }_{\text {rc,int }}$ is determined by metal-ligand binding interactions and the electrostatics among the metal, the protein environment, and the solvent. Whereas Clds have a proximal histidine with a pronounced imidazolate character and a mobile positively charged distal arginine, in coproheme-HemQ the basic character of the proximal histidine is less pronounced and no charged amino acid is found at the distal heme cavity in neighborhood of the heme iron. On the other hand, the higher negative charge of coproheme compared to that of heme $b$ distinguishes coproheme-HemQ from Cld (or heme $b$-HemQ) and could contribute to the selective enthalpic stabilization of the less charged ferric form (Table 2). Unfortunately, it was not possible to perform spectroelectrochemical studies of heme $b$-HemQ because the prosthetic group was partly lost during the redox reactions and the establishment of equilibria.

In any case, during transformation of coproheme to heme $b$, $\mathrm{p} 2$ and $\mathrm{p} 4$ are converted to vinyl groups and the H-bonds to G183 and Q187 (p2) as well as Y147 and S225 (p4) are lost. This loss of noncovalent interactions was nicely reflected by the significantly decreased thermal stability of heme $b$-HemQ compared to that of coproheme-HemQ. As a consequence, heme $b$ binding is significantly weakened and the reaction product can easily be extracted by another protein with a high affinity for heme $b$. Here we have probed this transfer with apomyoglobin that is known to bind the prosthetic group strongly with a $K_{\mathrm{D}}$ value in the low nanomolar range, ${ }^{60}$ which is $\sim 3$ 
orders of magnitude lower than the $K_{\mathrm{D}}$ value determined for heme $b$-HemQ. ${ }^{26}$

In conclusion, we show that apo-HemQs from Firmicutes rapidly and efficiently bind the substrate and redox cofactor coproheme in a biphasic reaction that results in the formation of a ferric high-spin resting state with a standard reduction potential around $-206 \mathrm{mV}$ versus the SHE. The $\alpha$-helix that carries the proximal ligand histidine seems to play a crucial role in capturing the substrate, which was reflected by the observed differences in its flexibility in the apoprotein and the loaded forms. Binding of coproheme significantly stabilizes the protein and restricts access to the distal heme cavity as suggested by the slower (but monophasic) binding of cyanide and the change in entropy during reduction of $\mathrm{Fe}$ (III) to $\mathrm{Fe}(\mathrm{II})$. Hydrogen peroxide, which is suggested to initiate the conversion of coproheme to heme $b$, should also have possibly restricted access to the heme cavity and react with the ferric protein, thereby converting the coproheme protein into a higher oxidation state, e.g., a high-valent iron intermediate. Because of the absence of catalytic amino acids at the distal side, it is not clear how the cleavage of $\mathrm{H}_{2} \mathrm{O}_{2}$ is catalyzed (homolytically vs heterolytically?) and how this oxidized enzyme intermediate subsequently mediates the decarboxylation reactions at $\mathrm{p} 2$ and p4. The possibility of releasing the intermediate monovinyl, monoproprionate deuteroheme followed by subsequent rebinding to HemQ (to allow decarboxylation at a single active site) is unlikely as MD simulations and heme transfer experiments did not suggest spontaneous release of either the substrate or the product. Celis et al. ${ }^{4}$ have reported that the reaction needs an amount of peroxide that is significantly greater than the expected amount of 2 equiv and that the conversions were often accompanied by heme bleaching and destruction. In any case, conversion of $\mathrm{p} 2$ and $\mathrm{p} 4$ to vinyl groups decreases the number of $\mathrm{H}$-bond and polar interactions with the protein and consequently the overall stability of heme $b$-HemQ. In addition, it increases the flexibility of the proximal heme cavity and the access channel. As a consequence, the reaction product can be released for incorporation into a target protein. Because of the importance of this enzyme for the survival of Firmicutes and Actinobacteria, ${ }^{2}$ it is reasonable to assume that the study of this protein will rapidly expand.

\section{ASSOCIATED CONTENT}

\section{S Supporting Information}

The Supporting Information is available free of charge on the ACS Publications website at DOI: 10.1021/acs.biochem.6b00701.

Data from simulation of the kinetics of binding of coproheme to apo-HemQ (Figure S1), binding of coproheme and heme $b$ to apo-myoglobin (Figure S2), transfer of heme to apo-myoglobin (Figure S3), thermal stability of LmHemQ (Figure S4), $\mathrm{pH}$ dependence of UV-vis absorption spectra of LmHemQ and SaHemQ (Figure S5), circular dichroism spectra of LmHemQ and SaHemQ (Figure S6), root-mean-square fluctuations of the protein backbones of HemQ (Figure S7), root-meansquare deviation and fluctuation of the protein backbone (Figure S8), accessibility to the coproheme/substrate binding site in apo- and coproheme-LmHemQ (Figure S9), and detailed listing of $\mathrm{H}$-bonds of coproheme propionates to the protein moiety derived from $\mathrm{MD}$ simulations (Table S1) (PDF)

\section{AUTHOR INFORMATION}

\section{Corresponding Author}

*E-mail: christian.obinger@boku.ac.at.

\section{Funding}

This project was supported by the Austrian Science Fund, FWF [Doctoral Program BioToP-Molecular Technology of Proteins (W1224) and Projects P25270, P29099, and I2429] and the Federal Ministry of Economy, Family and Youth through the initiative "Laura Bassi Centres of Expertise", funding the Center of Optimized Structural Studies ( $\mathrm{N}^{\circ}$ 253275).

\section{Notes}

The authors declare no competing financial interest.

\section{ABBREVIATIONS}

Cld, chlorite dismutase; coproheme, coproporphyrin III; DSC, differential scanning calorimetry; DyP, dye-decolorizing peroxidase; ECD, electronic circular dichroism; EPR, electron paramagnetic resonance; LmHemQ HemQ from L. monocytogenes; NdCld, chlorite dismutase from "Candidatus Nitrospira defluvii"; NwCld, chlorite dismutase from Nitrobacter winogradskyi; OTTLE, optical transparent thin-layer electrochemical; p2, propionate in coproheme at position $2 ; \mathrm{p} 4$, propionate in coproheme at position 4; p6, propionate in coproheme and heme $b$ at position $6 ; \mathrm{p} 7$, propionate in coproheme and heme $b$ at position 7; SaHemQ HemQ from $S$. aureus; rc, reaction; $\Delta H^{\circ}{ }_{\text {ro }}$ change in reaction enthalpy under standard conditions.

\section{REFERENCES}

(1) Dailey, H. A., Gerdes, S., Dailey, T. A., Burch, J. S., and Phillips, J. D. (2015) Noncanonical coproporphyrin-dependent bacterial heme biosynthesis pathway that does not use protoporphyrin. Proc. Natl. Acad. Sci. U. S. A. 112, 2210-2215.

(2) Dailey, H. A., and Gerdes, S. (2015) HemQ: An ironcoproporphyrin oxidative decarboxylase for protoheme synthesis in Firmicutes and Actinobacteria. Arch. Biochem. Biophys. 574, 27-35.

(3) Lobo, S. A., Scott, A., Videira, M. A., Winpenny, D., Gardner, M., Palmer, M. J., Schroeder, S., Lawrence, A. D., Parkinson, T., Warren, M. J., and Saraiva, L. M. (2015) Staphylococcus aureus haem biosynthesis: characterisation of the enzymes involved in final steps of the pathway. Mol. Microbiol. 97, 472-487.

(4) Celis, A. I., Streit, B. R., Moraski, G. C., Kant, R., Lash, T. D., Lukat-Rodgers, G. S., Rodgers, K. R., and DuBois, J. L. (2015) Unusual Peroxide-Dependent, Heme-Transforming Reaction Catalyzed by HemQ. Biochemistry 54, 4022-4032.

(5) Kostan, J., Sjöblom, B., Maixner, F., Mlynek, G., Furtmüller, P. G., Obinger, C., Wagner, M., Daims, H., and Djinović-Carugo, K. (2010) Structural and functional characterisation of the chlorite dismutase from the nitrite-oxidizing bacterium "Candidatus Nitrospira defluvii": identification of a catalytically important amino acid residue. J. Struct. Biol. 172, 331-342.

(6) Goblirsch, B., Kurker, R. C., Streit, B. R., Wilmot, C. M., and DuBois, J. L. (2011) Chlorite dismutases, DyPs, and EfeB: 3 microbial heme enzyme families comprise the CDE structural superfamily. $J$. Mol. Biol. 408, 379-398.

(7) Zámocký, M., Hofbauer, S., Schaffner, I., Gasselhuber, B., Nicolussi, A., Soudi, M., Pirker, K. F., Furtmüller, P. G., and Obinger, C. (2015) Independent evolution of four heme peroxidase superfamilies. Arch. Biochem. Biophys. 574, 108-119.

(8) van Ginkel, C. G., Rikken, G. B., Kroon, A. G., and Kengen, S. W. (1996) Purification and characterization of chlorite dismutase: a novel oxygen-generating enzyme. Arch. Microbiol. 166, 321-326.

(9) Lee, A. Q., Streit, B. R., Zdilla, M. J., Abu-Omar, M. M., and DuBois, J. L. (2008) Mechanism of and exquisite selectivity for O-O 
bond formation by the heme-dependent chlorite dismutase. Proc. Natl. Acad. Sci. U. S. A. 105, 15654-15659.

(10) Sugano, Y. (2009) DyP-type peroxidases comprise a novel heme peroxidase family. Cell. Mol. Life Sci. 66, 1387-1403.

(11) Yoshida, T., and Sugano, Y. (2015) A structural and functional perspective of DyP-type peroxidase family. Arch. Biochem. Biophys. 574, 49-55.

(12) Mlynek, G., Sjöblom, B., Kostan, J., Füreder, S., Maixner, F., Gysel, K., Furtmüller, P. G., Obinger, C., Wagner, M., Daims, H., and Djinović-Carugo, K. (2011) Unexpected diversity of chlorite dismutases: a catalytically efficient dimeric enzyme from Nitrobacter winogradskyi. J. Bacteriol. 193, 2408-2417.

(13) Goblirsch, B. R., Streit, B. R., Dubois, J. L., and Wilmot, C. M. (2010) Structural features promoting dioxygen production by Dechloromonas aromatica chlorite dismutase. J. Biol. Inorg. Chem. $15,879-888$.

(14) de Geus, D. C., Thomassen, E. A., Hagedoorn, P. L., Pannu, N. S., van Duijn, E., and Abrahams, J. P. (2009) Crystal structure of chlorite dismutase, a detoxifying enzyme producing molecular oxygen. J. Mol. Biol. 387, 192-206.

(15) Yoshida, T., Tsuge, H., Konno, H., Hisabori, T., and Sugano, Y. (2011) The catalytic mechanism of dye-decolorizing peroxidase DyP may require the swinging movement of an aspartic acid residue. FEBS J. 278, 2387-2394.

(16) Linde, D., Pogni, R., Canellas, M., Lucas, F., Guallar, V., Baratto, M. C., Sinicropi, A., Sáez-Jiménez, V., Coscolin, C., Romero, A., Medrano, F. J., Ruiz-Dueñas, F. J., and Martinez, A. T. (2015) Catalytic surface radical in dye-decolorizing peroxidase: A computational, spectroscopic and site-directed mutagenesis study. Biochem. J. 466, 253-262.

(17) Blanc, B., Mayfield, J. A., McDonald, C. A., Lukat-Rodgers, G. S., Rodgers, K. R., and DuBois, J. L. (2012) Understanding how the distal environment directs reactivity in chlorite dismutase: spectroscopy and reactivity of Arg183 mutants. Biochemistry 51, 1895-1910.

(18) Hofbauer, S., Gruber, C., Pirker, K. F., Sündermann, A., Schaffner, I., Jakopitsch, C., Oostenbrink, C., Furtmüller, P. G., and Obinger, C. (2014) Transiently produced hypochlorite is responsible for the irreversible inhibition of chlorite dismutase. Biochemistry 53, $3145-3157$.

(19) Schaffner, I., Hofbauer, S., Krutzler, M., Pirker, K. F., Furtmüller, P. G., and Obinger, C. (2015) Mechanism of chlorite degradation to chloride and dioxygen by the enzyme chlorite dismutase. Arch. Biochem. Biophys. 574, 18-26.

(20) Singh, R., Grigg, J. C., Armstrong, Z., Murphy, M. E., and Eltis, L. D. (2012) Distal heme pocket residues of B-type dye-decolorizing peroxidase: arginine but not aspartate is essential for peroxidase activity. J. Biol. Chem. 287, 10623-10630.

(21) Sugano, Y., Muramatsu, R., Ichiyanagi, A., Sato, T., and Shoda, M. (2007) DyP, a unique dye-decolorizing peroxidase, represents a novel heme peroxidase family: ASP171 replaces the distal histidine of classical peroxidases. J. Biol. Chem. 282, 36652-36658.

(22) Strittmatter, E., Liers, C., Ullrich, R., Wachter, S., Hofrichter, M., Plattner, D. A., and Piontek, K. (2013) First crystal structure of a fungal high-redox potential dye-decolorizing peroxidase: substrate interaction sites and long-range electron transfer. J. Biol. Chem. 288, 4095-4102.

(23) Singh, R, and Eltis, L. D. (2015) The multihued palette of dyedecolorizing peroxidases. Arch. Biochem. Biophys. 574, 56-65.

(24) Dailey, T. A., Boynton, T. O., Albetel, A. N., Gerdes, S., Johnson, M. K., and Dailey, H. A. (2010) Discovery and Characterization of HemQ: an essential heme biosynthetic pathway component. J. Biol. Chem. 285, 25978-25986.

(25) Mayfield, J. A., Hammer, N. D., Kurker, R. C., Chen, T. K., Ojha, S., Skaar, E. P., and DuBois, J. L. (2013) The chlorite dismutase (HemQ) from Staphylococcus aureus has a redox-sensitive heme and is associated with the small colony variant phenotype. J. Biol. Chem. 288, 23488-23504.

(26) Hofbauer, S., Hagmüller, A., Schaffner, I., Mlynek, G., Krutzler, M., Stadlmayr, G., Pirker, K. F., Obinger, C., Daims, H., Djinović-
Carugo, K., and Furtmüller, P. G. (2015) Structure and heme-binding properties of HemQ (chlorite dismutase-like protein) from Listeria monocytogenes. Arch. Biochem. Biophys. 574, 36-48.

(27) Hofbauer, S., Howes, B. D., Flego, N., Pirker, K. F., Schaffner, I., Mlynek, G., Djinovic-Carugo, K., Furtmüller, P. G., Smulevich, G., and Obinger, C. (2016) From chlorite dismutase towards HemQ-the role of the proximal H-bonding network in haeme binding. Biosci. Rep. 36, e00312.

(28) Sheldon, J. R., and Heinrichs, D. E. (2015) Recent developments in understanding the iron acquisition strategies of gram positive pathogens. FEMS Microbiol Rev. 39, 592-630.

(29) Edgar, R. C. (2004) MUSCLE: multiple sequence alignment with high accuracy and high throughput. Nucleic Acids Res. 32, 17921797.

(30) Tamura, K., Peterson, D., Peterson, N., Stecher, G., Nei, M., and Kumar, S. (2011) MEGA5: molecular evolutionary genetics analysis using maximum likelihood, evolutionary distance, and maximum parsimony methods. Mol. Biol. Evol. 28, 2731-2739.

(31) Teale, F. W. (1959) Cleavage of the haem-protein link by acid methylethylketone. Biochim. Biophys. Acta 35, 543.

(32) Le, P., Zhao, J., and Franzen, S. (2014) Correlation of heme binding affinity and enzyme kinetics of dehaloperoxidase. Biochemistry $53,6863-6877$.

(33) Stoll, S., and Schweiger, A. (2006) EasySpin, a comprehensive software package for spectral simulation and analysis in EPR. J. Magn. Reson. 178, 42-55.

(34) Peisach, J., Blumberg, W. E., Ogawa, S., Rachmilewitz, E. A., and Oltzik, R. (1971) The effects of protein conformation on the heme symmetry in high spin ferric heme proteins as studied by electron paramagnetic resonance. J. Biol. Chem. 246, 3342-3355.

(35) Aasa, R., and Vänngard, T. (1975) EPR signal intensity and powder shapes. Reexamination. J. Magn. Reson. 19, 308-315.

(36) Battistuzzi, G., Borsari, M., Ranieri, A., and Sola, M. (2002) Redox thermodynamics of the $\mathrm{Fe}(3+) / \mathrm{Fe}(2+)$ couple in horseradish peroxidase and its cyanide complex. J. Am. Chem. Soc. 124, 26-27.

(37) Battistuzzi, G., D’Onofrio, M., Borsari, M., Sola, M., Macedo, A. L., Moura, J. J., and Rodrigues, P. (2000) Redox thermodynamics of low-potential iron-sulfur proteins. J. Biol. Inorg. Chem. 5, 748-760.

(38) Varadarajan, R., Zewert, T. E., Gray, H. B., and Boxer, S. G. (1989) Effects of buried ionizable amino acids on the reduction potential of recombinant myoglobin. Science 243, 69-72.

(39) Schmid, N., Eichenberger, A. P., Choutko, A., Riniker, S., Winger, M., Mark, A. E., and van Gunsteren, W. F. (2011) Definition and testing of the GROMOS force-field versions 54A7 and 54B7. Eur. Biophys. J. 40, 843-856.

(40) Schmid, N., Christ, C., Christen, M., Eichenberger, A., and van Gunsteren, W. (2012) Architecture, implementation and parallelisation of the GROMOS software for biomolecular simulation. Comput. Phys. Commun. 183, 890-903.

(41) Zou, C., Larisika, M., Nagy, G., Srajer, J., Oostenbrink, C., Chen, X., Knoll, W., Liedberg, B., and Nowak, C. (2013) Two-Dimensional Heterospectral Correlation Analysis of the Redox-Induced Conformational Transition in Cytochrome c Using Surface-Enhanced Raman and Infrared Absorption Spectroscopies on a Two-Layer Gold Surface. J. Phys. Chem. B 117, 9606-9614.

(42) Berendsen, H. J. C., Postma, J. P. M., van Gunsteren, W. F., and Hermans, J. (1981) Interaction models for water in relation to protein hydration, Reidel, Dordrecht, The Netherlands.

(43) Tironi, I. G., Sperb, R., Smith, P. E., and van Gunsteren, W. F. (1995) A generalized reaction field method for molecular-dynamics simulations. J. Chem. Phys. 102, 5451-5459.

(44) Heinz, T. N., van Gunsteren, W. F., and Hünenberger, P. H. (2001) Comparison of four methods to compute the dielectric permittivity of liquids from molecular dynamics simulations. J. Chem. Phys. 115, 1125-1136.

(45) Berendsen, H. J. C., Postma, J. P. M., van Gunsteren, W. F., DiNola, A., and Haak, J. R. (1984) Molecular-dynamics with coupling to an external bath. J. Chem. Phys. 81, 3684-3690. 
(46) Ryckaert, J. P., Ciccotti, G., and Berendsen, H. J. C. (1977) Numerical integration of cartesian equations of motion of a system with constraints: Molecular dynamics of n-alkanes. J. Comput. Phys. 23, 327-341.

(47) Eichenberger, A. P., Allison, J. R., Dolenc, J., Geerke, D. P., Horta, B. A., Meier, K., Oostenbrink, C., Schmid, N., Steiner, D., Wang, D., and van Gunsteren, W. F. (2011) GROMOS++ Software for the Analysis of Biomolecular Simulation Trajectories. J. Chem. Theory Comput. 7, 3379-3390.

(48) Deniau, C., Gilli, R., Izadi-Pruneyre, N., Létoffé, S., Delepierre, M., Wandersman, C., Briand, C., and Lecroisey, A. (2003) Thermodynamics of heme binding to the HasA(SM) hemophore: effect of mutations at three key residues for heme uptake. Biochemistry 42, 10627-10633.

(49) Hofbauer, S., Gysel, K., Mlynek, G., Kostan, J., Hagmüller, A., Daims, H., Furtmüller, P. G., Djinović-Carugo, K., and Obinger, C. (2012) Impact of subunit and oligomeric structure on the thermal and conformational stability of chlorite dismutases. Biochim. Biophys. Acta, Proteins Proteomics 1824, 1031-1038.

(50) Kelly, S. M., Jess, T. J., and Price, N. C. (2005) How to study proteins by circular dichroism. Biochim. Biophys. Acta, Proteins Proteomics 1751, 119-139.

(51) Hofbauer, S., Bellei, M., Sündermann, A., Pirker, K. F., Hagmüller, A., Mlynek, G., Kostan, J., Daims, H., Furtmüller, P. G., Djinovic-Carugo, K., Oostenbrink, C., Battistuzzi, G., and Obinger, C. (2012) Redox Thermodynamics of High-Spin and Low-Spin Forms of Chlorite Dismutases with Diverse Subunit and Oligomeric Structures. Biochemistry 51, 9501-9512.

(52) Dailey, H. A., Wu, C. K., Horanyi, P., Medlock, A. E., NajahiMissaoui, W., Burden, A. E., Dailey, T. A., and Rose, J. (2007) Altered orientation of active site residues in variants of human ferrochelatase. Evidence for a hydrogen bond network involved in catalysis. Biochemistry 46, 7973-7979.

(53) Kabsch, W., and Sander, C. (1983) Dictionary of protein secondary structure: pattern recognition of hydrogen-bonded and geometrical features. Biopolymers 22, 2577-2637.

(54) De Schutter, A., Correia, H. D., Freire, D. M., Rivas, M. G., Rizzi, A., Santos-Silva, T., González, P. J., and Van Doorslaer, S. (2015) Ligand Binding to Chlorite Dismutase from Magnetospirillum sp. J. Phys. Chem. B 119, 13859-13869.

(55) Lambeth, J. D., and Kamin, H. (1979) Adrenodoxin reductase.adrenodoxin complex. Flavin to iron-sulfur electron transfer as the rate-limiting step in the $\mathrm{NADPH}$-cytochrome $\mathrm{c}$ reductase reaction. J. Biol. Chem. 254, 2766-2774.

(56) Battistuzzi, G., Bellei, M., Bortolotti, C. A., and Sola, M. (2010) Redox properties of heme peroxidases. Arch. Biochem. Biophys. 500, 21-36.

(57) Bellei, M., Jakopitsch, C., Battistuzzi, G., Sola, M., and Obinger, C. (2006) Redox thermodynamics of the ferric-ferrous couple of wildtype synechocystis KatG and $\mathrm{KatG}(\mathrm{Y} 249 \mathrm{~F})$. Biochemistry 45, 47684774.

(58) Battistuzzi, G., Bellei, M., Zederbauer, M., Furtmüller, P. G., Sola, M., and Obinger, C. (2006) Redox thermodynamics of the $\mathrm{Fe}(\mathrm{III}) / \mathrm{Fe}$ (II) couple of human myeloperoxidase in its high-spin and low-spin forms. Biochemistry 45, 12750-12755.

(59) Battistuzzi, G., Bellei, M., Borsari, M., Di Rocco, G., Ranieri, A., and Sola, M. (2005) Axial ligation and polypeptide matrix effects on the reduction potential of heme proteins probed on their cyanide adducts. J. Biol. Inorg. Chem. 10, 643-651.

(60) Gryczynski, Z., Lubkowski, J., and Bucci, E. (1995) Hemeprotein interactions in horse heart myoglobin at neutral $\mathrm{pH}$ and exposed to acid investigated by time-resolved fluorescence in the picoto nanosecond time range. J. Biol. Chem. 270, 19232-19237. 